\title{
Non-spherical core collapse supernovae
}

\section{The late-time evolution of globally anisotropic neutrino-driven explosions and their implications for SN $1987 A^{\star}$}

\author{
K. Kifonidis ${ }^{1}$, T. Plewa ${ }^{2}$, L. Scheck ${ }^{1}$, H.-Th. Janka ${ }^{1}$, and E. Müller ${ }^{1}$ \\ 1 Max-Planck-Institut für Astrophysik, Karl-Schwarzschild-Straße 1, 85741 Garching, Germany \\ e-mail: kok@mpa-garching.mpg.de \\ 2 Center for Astrophysical Thermonuclear Flashes, University of Chicago, 5640 S. Ellis Avenue, Chicago, IL 60637, USA
}

Received 12 November 2005 / Accepted 20 February 2006

\begin{abstract}
Two-dimensional simulations of strongly anisotropic supernova explosions of a nonrotating $15 M_{\odot}$ blue supergiant progenitor are presented, which follow the hydrodynamic evolution from times shortly after shock formation until hours later. It is shown that explosions which around the time of shock revival are dominated by low-order unstable modes (i.e. by a superposition of the $l=2$ and $l=1$ modes, in which the former is strongest), are consistent with all major observational features of SN $1987 \mathrm{~A}$, in contrast to models which show high-order mode perturbations only and were published in earlier work. Among other items, the low-mode models exhibit final iron-group velocities of up to $\sim 3300 \mathrm{~km} \mathrm{~s}^{-1}$, strong mixing at the $\mathrm{He} / \mathrm{H}$ composition interface, with hydrogen being mixed downward in velocity space to only $500 \mathrm{~km} \mathrm{~s}^{-1}$, and a final prolate anisotropy of the inner ejecta with a major to minor axis ratio of about 1.6. The success of low-mode explosions with an energy of about $2 \times 10^{51} \mathrm{erg}$ to reproduce these observed features is based on two effects: the (by $40 \%$ ) larger initial maximum velocities of metal-rich clumps compared to our high-mode models, and the initial global deformation of the shock. The first effect protects the (fastest) clumps from interacting with the strong reverse shock that forms below the $\mathrm{He} / \mathrm{H}$ composition interface, by keeping their propagation timescale through the He-core shorter than the reverse shock formation time. This ensures that the outward motion of the clumps remains always subsonic, and that thus their energy dissipation is minimal (in contrast to the supersonic case). The second effect is responsible for the strong inward mixing of hydrogen: the aspherical shock deposits large amounts of vorticity into the $\mathrm{He} / \mathrm{H}$ interface layer at early times (around $t=100 \mathrm{~s}$ ). This triggers the growth of a strong Richtmyer-Meshkov instability that results in a global anisotropy of the inner ejecta at late times (i.e. around $t=10000 \mathrm{~s}$ ), although the shock itself has long become spherical by then. The simulations suggest a coherent picture, which explains the observational data of SN 1987 A within the framework of the neutrino-driven explosion mechanism using a minimal set of assumptions. It is therefore argued that other paradigms, which are based on (more) controversial physics, may not be required to explain this event.
\end{abstract}

Key words. hydrodynamics - instabilities - nucleosynthesis - shock waves - supernovae: general

\section{Introduction}

Large-scale anisotropies and mixing, as well as smaller-scale clumping, are common features of supernova $(\mathrm{SN})$ remnants like Cas A, or SN $1987 \mathrm{~A}$, and have been attributed to the occurrence of hydrodynamic instabilities (in particular the Rayleigh-Taylor or RT instability) in the SN explosions that gave rise to these remnants. Starting the supernova by depositing kinetic and/or thermal energy into a progenitor model, many groups in the past have performed multidimensional hydrodynamic simulations to study the mixing associated with these effects. However, so far the models have not been able to explain the observed features in a satisfactory way. An extensive bibliography and overview of such work was given in the first paper of the present series (Kifonidis et al. 2003, henceforth Paper I).

In this latter reference we ourselves have embarked on a somewhat more ambitious effort, namely to investigate the observational consequences of the modern (i.e. multidimensional, or convectively supported) neutrino-driven explosion paradigm

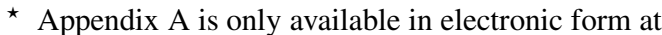
http://www. edpsciences.org for core collapse supernovae. For this purpose we performed an exploratory, high-resolution 2D simulation of a type II supernova in a $15 M_{\odot}$ blue supergiant which included a detailed modelling of the explosion itself, and which we dubbed "Model T310a". This calculation covered the time from $20 \mathrm{~ms}$ up to more than $5 \mathrm{~h}$ after core bounce, and, at the time of submission of the present paper, is still the only multidimensional simulation that followed the hydrodynamic evolution and the growth of anisotropies from the earliest moments after core collapse until well after the time of shock emergence from the stellar photosphere. In this way the effects of neutrino heating, convection, and explosive nucleosynthesis deep inside the core, as well as their impact on the Rayleigh-Taylor instabilities in the stellar envelope were all taken into account.

For simulating the onset of the explosion in Paper I, we approximated the neutrino transport by a simple light-bulb scheme, and parametrized the neutrino fluxes emitted by the optically thick proto-neutron star core. In an attempt to reproduce the high nickel velocities of SN 1987 A, the most sensitive test case for supernova theory available to date, we performed the calculation using rather high initial values for the neutrino fluxes, and a subsequent exponential decay of these fluxes with time. This 
prescription initially appeared promising, since it gave a rather energetic explosion. However, the maximum nickel velocities that were finally obtained from the simulation, were disappointingly small.

Furthermore, because the explosion in this model set in very rapidly, it did not allow hydrodynamic instabilities, which occur in the neutrino-heated post-shock layers, to grow to sufficient strength such that they could deform the shock. Hence the model showed only relatively small-scale deviations from spherical symmetry - i.e., higher order modes of the fluid flow in terms of an expansion of the inhomogeneities in Legendre polynomials of order $l$ - and was not able to reproduce the global anisotropy of the ejecta and the extent of the mixing observed in SN 1987 A. It also left behind a small neutron star of only about $1.1 M_{\odot}$ (baryonic). Apparently, our parametrization of the neutrino fluxes had resulted in too short an explosion timescale, and our hope was that the aforementioned problems might be overcome with an improved description of the neutrino effects, e.g. by a modification of the ( $\mathrm{ad} \mathrm{hoc}$ ) assumptions on the behavior of the boundary conditions for the neutrino parameters.

This provided the motivation for the systematic twodimensional neutrino-hydrodynamic parameter study that we presented in Scheck et al. $(2004,2006)$. In these works, we replaced the high initial values of the core neutrino fluxes with lower ones, and the (old) exponential decay with a much slower decline over time, an assumption which is in agreement with the results of current simulations employing Boltzmann neutrino transport. (Note that the core luminosity is imposed at our inner grid boundary which follows a Lagrangian mass coordinate.) Furthermore, we dropped the simple neutrino light bulb approximation and replaced it with a new, grey, characteristics-based neutrino transport scheme that we use in the free-streaming and semi-transparent regimes. This allows us to take into account the effects of neutrino heating and cooling on the flux, e.g. to include the effect of mass accretion on the neutrino luminosities (for details see Scheck et al. 2006).

With this new approach Scheck et al. (2004, 2006) were able to demonstrate that, if the explosion sets in sufficiently slowly, low-mode hydrodynamic instabilities, i.e. low-mode convection (Chandrasekhar 1961; Herant 1995) in the neutrinoheating layer, and the recently discovered advective-acoustic (Foglizzo \& Tagger 2000; Foglizzo 2002; Foglizzo \& Galetti 2003; Foglizzo et al. 2005; Ohnishi et al. 2005), and SASI instabilities (Blondin et al. 2003; Blondin \& Mezzacappa 2005), can grow from small random perturbations behind the shock and result in a global anisotropy of the shock and the ejecta. Moreover such explosions with typical supernova energies also leave behind neutron stars with reasonable masses and high recoil velocities, all at the same time. Scheck et al. (2006) furthermore showed that the occurrence of different low-order unstable modes $(l=1, l=2)$ in the post-shock flow, might even explain a possible bimodality of the observed galactic pulsar velocity distribution without the need to assume any physics in addition to what is already part of the neutrino-driven explosion paradigm.

Yet, it needs to be pointed out that currently the viability of neutrino-driven supernova explosions for progenitor stars of more than $\sim 10 M_{\odot}$ is still an open question (see, e.g., Buras et al. 2003, 2006a,b). Our adherence to the neutrino-driven explosion mechanism and our procedure of triggering supernova explosions by the use of a boundary condition for the neutrino flux may be justified by the fact that uncertainties are still present even in the most sophisticated recent 2D supernova simulations, despite the significant improvements in the treatment of neutrino physics and transport that have been achieved compared to the first generation of multidimensional modelling. Such recent 2D simulations, which include spectral neutrino transport, (see Buras et al. 2003, 2006a,b) could not confirm the success of first generation models, which employed gray neutrino diffusion and found explosions (e.g. Herant et al. 1994; Burrows et al. 1995; Fryer 1999; Fryer \& Warren 2002, 2004).

However, it should be noted that 3D simulations with spectral transport have not been performed yet, and might reveal important differences in the flow dynamics compared to axisymmetric models, in particular with respect to the growth of convective and Rayleigh-Taylor instabilities, non-radial accretion shock instabilities, and vortex behaviour. Moreover, the question whether convective or mixing processes below and around the neutrinosphere could enhance the neutrinospheric neutrino emission and thus support the neutrino heating behind the shock, must still be considered as unsettled: Doubly diffusive instabilities (Bruenn et al. 2006), neutrino-bubble instabilities (Socrates et al. 2005), or magnetic buoyancy instabilities (Wilson et al. 2005) deserve further investigation by multidimensional modelling. Also magneto-rotational instabilities were suggested to aid the explosion by creating viscous heating behind the shock in addition to the still dominant energy input there by neutrinos (Thompson et al. 2005).

Although these currently unresolved issues need to be kept in mind, the (2D) simulations of Scheck et al. (2006) suggest a quite remarkable perspective, namely that many fundamental properties of observed supernovae and neutron stars might be traced back to the same origin, i.e. to non-radial hydrodynamic instabilities during the first second of a neutrino-driven SN explosion. However, as these calculations did not cover also the later phases of the explosion, they could not provide insight into the inevitable interaction of the inner ejecta with the (possibly massive, e.g. hydrogen) envelope of the progenitor. Since this interaction (which happens on a timescale of hours to days, depending on the type of the progenitor) determines many of the more intricate observational features, especially of type II supernovae, an important question still remains unanswered: can globally anisotropic neutrino-driven explosions also lead to high final nickel velocities, strong mixing at the $\mathrm{He} / \mathrm{H}$ composition interface, large final ejecta anisotropies, and hence sizeable polarization of the emitted light?

A positive answer to this question would not only offer a (natural) explanation for the gamut of observational data collected in case of SN $1987 \mathrm{~A}$, or SN remnants like Cas A. It would also provide substantial support to the neutrino mechanism. The present paper is an attempt to explore this question in the framework of two-dimensional, i.e. axisymmetric simulations. We exemplarily follow the evolution of globally anisotropic explosions of the type studied in Scheck et al. (2004, 2006) from several milliseconds up to several hours after core bounce in high-resolution simulations.

Although we do not simulate the "full SN problem", but trigger the neutrino-driven explosion by the use of a suitable inner boundary condition, we nevertheless think that our modelling approach is adequate to address the aforementioned points because it is "consistent" in the following sense: the distribution of kinetic and internal energy in the flow, the number and size of flow structures due to hydrodynamic instabilities, the shape and asymmetry of the supernova shock, and the distribution of nucleosynthetic products (both in real and velocity space) develop naturally in a detailed neutrino-hydrodynamic calculation, in response to a boundary condition for the neutrino flux and the contraction of the neutron star core. Neither of the former quantities, which determine the kind of observables that we are 
interested in, is put in "by hand" - in contrast to previous work in which ad hoc prescriptions were used for at least one of these aspects.

The question of how close our boundary condition and initiation of the explosion come to reality cannot be finally assessed at present, because of the lack of detailed observed neutrino light curves, and/or the aforementioned uncertainties inherent to more sophisticated modelling of the (neutrino-driven) explosion mechanism. Yet, the choice of particular parameter values for this boundary condition affects mainly the integral characteristics of the explosion like its total energy and time of onset, as well as the integral properties of the neutron star, e.g. its mass and contraction time scale (see again Scheck et al. 2006 for details). A particular parametrization is in the end vindicated by its success to reproduce the observational features of SN $1987 \mathrm{~A}$.

The stellar model that we shall consider is the $15 M_{\odot}$ blue supergiant SN 1987 A progenitor of Woosley et al. (1988), which we already used in Paper I. This makes the present work a direct sequel and extension of this earlier investigation. It is our hope that these papers, together with the constraints obtained from observations, will serve to elucidate the still poorly understood hydrodynamics of SN 1987 A, and help to disclose the true nature of this and similar events.

We shall proceed with a description of the most recent modifications to our computer codes in Sect. 2. We then comment briefly on our initial data and some computational aspects in Sect. 3. The results of our calculations are presented in Sect. 4, and compared with SN 1987 A in Sect. 5. Our conclusions can be found in Sect. 6. The Appendix finally contains a summary of the AUSM+ numerical flux function of Liou (1996) that is used in one of our hydrodynamics codes.

\section{Modifications to the codes}

The computer programs that were used for the present calculations, are the neutrino hydrodynamics code of Scheck et al. $(2004,2006)$ and the version of the adaptive mesh refinement hydrodynamics code AMRA that we described in Paper I. Both use the direct Eulerian version of the Piecewise Parabolic Method (PPM) of Colella \& Woodward (1984) to integrate the hydrodynamics equations. Several changes were made to the former code, though. In particular we have improved its potential to resolve shocks, by updating the hybrid Riemann solver algorithm which we employed in Scheck et al. (2004, 2006). In these works we have made use of the standard (in PPM) exact Riemann solver to evolve all zones on the grid, except for those zones which are located in the vicinity of strong (grid-aligned) shocks, for which the HLLE flux of Einfeldt (1988) was employed (cf. Paper I). After replacing the HLLE flux by the AUSM+ flux of Liou (1996) (see also Appendix A) we now achieve both, postshock states completely free of odd-even decoupling, and very sharp shocks with only one or two interior points (while with the HLLE flux at least 3-4 points are required).

We have also completely revised the nucleosynthesis modules of this code (see the end of this section for our motivation). For zones with temperatures $1.5 \times 10^{9} \mathrm{~K}<T<T_{\max }^{\text {net }}$ we now solve a 32 species nuclear reaction network instead of the modified $\alpha$-nuclei network described in Paper I. The isotopes considered in the 32 species network are listed in Table 1. Nuclear data and rates for 141 reactions in total stem from the compilation of Thielemann (see Thielemann et al. 1996; Hoffman et al. 1999, and the references therein). The ordinary differential equations (ODEs) constituting the nuclear network are solved with the
Table 1. Isotopes of the 32 species nuclear reaction network.

\begin{tabular}{cccccccc}
\hline \hline $\mathrm{n}$ & $\mathrm{p}$ & ${ }^{4} \mathrm{He}$ & ${ }^{12} \mathrm{C}$ & ${ }^{16} \mathrm{O}$ & ${ }^{20} \mathrm{Ne}$ & ${ }^{24} \mathrm{Mg}$ & ${ }^{28} \mathrm{Si}$ \\
${ }^{32} \mathrm{~S}$ & ${ }^{36} \mathrm{Ar}$ & ${ }^{40} \mathrm{Ca}$ & ${ }^{44} \mathrm{Ti}$ & ${ }^{50} \mathrm{Ti}$ & ${ }^{48} \mathrm{Cr}$ & ${ }^{54} \mathrm{Cr}$ & ${ }^{55} \mathrm{Cr}$ \\
${ }^{54} \mathrm{Mn}$ & ${ }^{55} \mathrm{Mn}$ & ${ }^{56} \mathrm{Mn}$ & ${ }^{52} \mathrm{Fe}$ & ${ }^{54} \mathrm{Fe}$ & ${ }^{55} \mathrm{Fe}$ & ${ }^{56} \mathrm{Fe}$ & ${ }^{57} \mathrm{Fe}$ \\
${ }^{58} \mathrm{Fe}$ & ${ }^{55} \mathrm{Co}$ & ${ }^{56} \mathrm{Co}$ & ${ }^{56} \mathrm{Ni}$ & ${ }^{57} \mathrm{Ni}$ & ${ }^{58} \mathrm{Ni}$ & ${ }^{60} \mathrm{Ni}$ & ${ }^{60} \mathrm{Zn}$ \\
\hline
\end{tabular}

variable-order Bader-Deuflhard semi-implicit, stiff ODE integrator, which employs sub-stepping and Richardson extrapolation to achieve very large effective (time) steps (Bader \& Deuflhard 1983; Press et al. 1992; Timmes 1999).

For $T>T_{\max }^{\text {net }}$ a nuclear statistical equilibrium (NSE) solution is computed: given the temperature, $T$, density, $\rho$, electron fraction, $Y_{\mathrm{e}}$, and the parameters $Z_{i}$ (charge number), $A_{i}$ (mass number), $B_{i}$ (binding energy) and $G_{i}(T)$ (partition function) of species $i$, we iteratively solve (with a globally convergent multidimensional Newton-Raphson scheme) the Saha equations

$Y_{i}=\left(\rho N_{A}\right)^{A_{i}-1} \frac{G_{i}}{2^{A_{i}}} A_{i}^{3 / 2}\left(\frac{2 \pi \hbar^{2}}{m_{u} k T}\right)^{\frac{3}{2}\left(A_{i}-1\right)} \exp \left(B_{i} / k T\right) Y_{\mathrm{n}}^{\left(A_{i}-Z_{i}\right)} Y_{\mathrm{p}}^{Z_{i}}$

and the equations of charge and mass conservation

$\sum_{i} Z_{i} Y_{i}=Y_{\mathrm{e}}, \quad \sum_{i} A_{i} Y_{i}=1$

for the neutron and proton abundances, $Y_{\mathrm{n}}$ and $Y_{\mathrm{p}}$, respectively. From these follow the abundances $Y_{i}$ of all other species by virtue of Eq. (1).

Nuclear screening was included in the NSE solution according to Hix \& Thielemann (1996), but is currently neglected in the nuclear network solver. In order to get a perfect match between the numerical solution of the nuclear network ODEs and the NSE solution we had to set $T_{\max }^{\text {net }}=8 \times 10^{9} \mathrm{~K}$, i.e. we use the nuclear network well into the medium-temperature NSE regime, where the composition consists of neutrons, protons, and $\alpha$-particles, but essentially no heavy nuclei. The resulting algorithm proved to be very robust and shows no difficulties in making smooth transitions from temperatures as high as $\sim 10^{11} \mathrm{~K}$ to less than $10^{9} \mathrm{~K}$, and vice-versa. In addition, it turned out to be very efficient. Only a small number of (main) steps per burning zone are necessary with the Bader-Deuflhard method to obtain a relative integration accuracy of $10^{-4}$. The employed $T_{\max }^{\text {net }}$ turned out to be still low enough to avoid the reaction rates becoming extremely large, and hence nuclear timescales becoming extremely small ${ }^{1}$.

The coupling of the nuclear burning algorithm with the hydrodynamics was done in the same operator-split, "coprocessing" approximation that we described in Paper I, i.e. energy and composition changes from the 32 species solution are currently not fed back into the solution of the hydrodynamics, which instead still relies on the smaller 4-species NSE solution tabulated in the equation of state of Janka \& Müller (1996). Work is in progress to extend the implicit nuclear algorithm with an energy equation (see Müller 1986), and with the leptonic equation of state of Timmes \& Swesty (2000), in order to obtain a fully coupled treatment of the composition changes, nuclear source terms, equation of state, and hydrodynamics in future simulations.

\footnotetext{
1 Despite our efficient solution of the 32-species network, the simulations still require about a factor of five more computer time than without computing the nucleosynthesis.
} 
We note here that our primary motivation for coding the present method was its enhanced robustness and efficiency over the implementation of the nuclear physics that we used in Paper I. This enables us to handle situations in which the previous algorithm (wich was based on the "classical" first-order Euler backward time discretization), either experienced convergence problems or required too many nuclear time steps to cover a hydrodynamic step. As a side effect, and due to the large gain in efficiency provided by the Bader-Deuflhard integrator (see Timmes 1999), we can now also solve larger reaction networks. However, improvements in the accuracy of the nuclear yields which may thereby be achieved, need to be contrasted with the fact that the relative yields of many isotopes within the iron group (e.g. the ${ }^{56} \mathrm{Ni} /{ }^{58} \mathrm{Fe}$ ratio), depend extremely sensitively on the electron fraction, $Y_{\mathrm{e}}$, and thus require also a more sophisticated, non-grey neutrino transport scheme for their accurate computation than the neutrino treatment we employ here (see also the discussion of these issues in Paper I). For this reason we will give only integrated abundances (or mass fractions) for the iron group in this paper, instead of individual abundances for single iron-group nuclei.

\section{Computational aspects}

\subsection{Numerical mesh, initial, and boundary conditions}

Throughout this work, 2D spherical coordinates $(r, \vartheta)$, a computational grid with $180^{\circ}$ width in $\vartheta$, and axisymmetry are adopted. To perform the present simulations we chose to stick to the same $15 M_{\odot}$ blue supergiant model of Woosley et al. (1988) and the post-bounce model of Bruenn (1993) (at a time of $20 \mathrm{~ms}$ after core bounce) that we have already used as initial data in Paper I.

Similar to this latter work, we follow the first second of the explosion using neutrino-hydrodynamics calculations. These are set up as described in Scheck et al. (2006) and we refer the reader to this paper for details, in particular for the employed boundary conditions.

We wish to highlight one point though, which is connected to the fact that we start our 2D simulations from supernova progenitor and post-bounce models that are one-dimensional. Unlike the grid-less smooth particle hydrodynamics (SPH) method, our grid-based Eulerian hydrodynamics codes exhibit such a low level of numerical noise that a one-dimensional, isotropic initial configuration remains perfectly isotropic when evolved on a $2 \mathrm{D}$ grid, even if, e.g., a stratification is present in the 1D initial data which is prone to become convective. To trigger the growth of non-radial hydrodynamic instabilities in the post-shock flow, we thus need to explicitly apply a small random perturbation to the initial model, which we add to the velocity field and for which we typically use an amplitude of $0.1 \%$. This perturbation is applied only once, at the beginning of the calculation, i.e. at $t \approx 20 \mathrm{~ms}$ post-bounce.

To prescribe the neutrino radiation field of the contracting neutron star core we make use of the same inner boundary contraction and parametrization (with $\Delta E_{\text {core }}^{\infty}=0.18 M_{\odot} c^{2}, t_{L}=$ $1.0 \mathrm{~s}$ ) that was employed for model B18 in Scheck et al. (2006). This resulted in a total electron flavour neutrino luminosity of the neutron star core, $L^{\text {core }}=L_{v_{\mathrm{e}}}+L_{\bar{v}_{\mathrm{e}}}=4.45 \times 10^{52} \mathrm{erg} / \mathrm{s}$, which was assumed to be constant during the first second.

In order to explore different explosion asymmetries resulting from the non-radial instabilities, we calculated two models with this particular setup that differ only in the initial perturbation. To achieve this we simply used two different random number sequences to perturb the initial velocity field in these two
Table 2. Models for the Woosley et al. (1988) blue supergiant. Given are the explosion timescale, $t_{\mathrm{exp}}$, and the entire simulated time, $t_{\mathrm{sim}}$, as well as the explosion energy at a time of $3000 \mathrm{~s}, E_{\exp }^{t=3000 \mathrm{~s}}$, and the baryonic mass of the neutron star at $t \approx 1 \mathrm{~s}$ (without fallback). The explosion timescale is defined by the time after core bounce when the integral of the total energy over all grid cells with positive values of that quantity and positive radial velocities, is larger than $10^{48} \mathrm{erg}$.

\begin{tabular}{lcccc}
\hline \hline Model & $\begin{array}{c}t_{\text {exp }} \\
{[\mathrm{s}]}\end{array}$ & $\begin{array}{c}E_{\text {exp }}^{t=3000 \mathrm{~s}} \\
{\left[10^{51} \mathrm{erg}\right]}\end{array}$ & $\begin{array}{c}M_{\mathrm{ns}} \\
{\left[M_{\odot}\right]}\end{array}$ & $\begin{array}{c}t_{\text {sim }} \\
{[\mathrm{s}]}\end{array}$ \\
\hline b18a (disregarded) & 0.190 & 1.0 & 1.3 & $10^{4}$ \\
b18b & 0.185 & 1.0 & 1.3 & $2 \times 10^{4}$ \\
b23a & 0.138 & 2.0 & 1.2 & $2 \times 10^{4}$ \\
\hline T310a (Paper I) & 0.062 & 1.7 & 1.1 & $2 \times 10^{4}$ \\
\hline
\end{tabular}

runs. Everything else, in particular the perturbation amplitude that we employed, was kept the same. The resulting models will henceforth be called b18a and b18b. We point out here - and elaborate on this in more detail in Scheck et al. (2006) - that already such minor differences between the simulations produce different ejecta asymmetries, because the growth of the initial perturbations is extremely nonlinear. In fact it is chaotic.

We also used a second parametrization for the inner boundary condition which differs from the former setup only in a somewhat larger gravitational binding energy loss of the neutron star core, $\Delta E_{\text {core }}^{\infty}=0.23 M_{\odot} c^{2}$. This leads to an $L^{\text {core }}$ which is by $28 \%$ higher than in the b18 models, and gives rise to model b23a, which attained an explosion energy that is twice as large as that of the b18 case (see Table 2 and Sect. 4.1).

Note that throughout this paper we use lower-case initial letters in our model names to distinguish the present simulations from the models of Scheck et al. (2006), which did not make use of the nucleosynthesis solver, and were still performed using the HLLE flux instead of the AUSM+ flux (see above). Note also that in all of our simulations (which were carried out with 400 radial and 180 angular zones), the inner boundary condition for neutrinos was assumed to be isotropic, and that the core luminosities were held constant over the time $t_{L}$ (for a motivation and full details, including a comparison with results from Boltzmann transport calculations, see Scheck et al. 2006).

The hydrodynamic evolution after the first second (which includes the growth of Rayleigh-Taylor and Richtmyer-Meshkov instabilities) was simulated with the AMRA code and the mesh refinement hierarchy, mesh resolution, remapping scheme, and boundary conditions that were described in Paper I. This allows us to temporarily achieve a resolution equivalent to covering the entire progenitor with $2.6 \times 10^{6}$ equidistant radial zones, although the effective number of radial zones on the finest level of the AMR grid hierarchy was only 3072. The effective number of angular zones on this refinement level was 768 .

\subsection{Constraints due to the grid-geometry}

At the outset we must acknowledge that a two-dimensional hydrodynamic study in standard spherical coordinates has the drawback of messing with the well-known coordinate singularity at the poles of the spherical grid. From earlier calculations of the Rayleigh-Taylor mixing in core collapse supernovae employing spherical coordinates it is known (see Kane et al. 2000, and Paper I) that Rayleigh-Taylor fingers grow faster along the poles than in other regions of the grid, and may even evolve to (artificial) "axial jets" if the simulation is followed to late times. 
This effect is most likely enhanced by the imposed axisymmetry, which restricts the degrees of freedom of the flow, and promotes its convergence along the impenetrable poles.

If the initial conditions do not deviate too strongly from spherical symmetry, the axial artifacts remain rather localized, and do not affect the global character of the solution (see the calculations of Paper I, and Kane et al. 2000). However, this may no longer be the case if one starts from very anisotropic situations. To illustrate this, we show the distributions of entropy and density at $t \approx 1 \mathrm{~s}$ and at $t \approx 3000 \mathrm{~s}$, respectively, for models b18a, b18b, and b23a in Fig. 1. Of these models the first one (Model b18a) is the most anisotropic, being dominated by the $l=1$ (bipolar) mode one second after core bounce, while the latter two show a dominance of the $l=2$ (quadrupole) mode (with a smaller $l=1$ contribution).

It is apparent from Figs. 1d-f that in all of the models the solution at a time of $\sim 3000 \mathrm{~s}$ is affected significantly in the polar regions, showing a pair of polar "bulges" whose origin is in all cases a pair of polar Rayleigh-Taylor fingers. These expand with much higher velocity than the surrounding material and ultimately deform the main shock.

Yet, while in models b18b and b23a the solution is still undisturbed at distances $\gtrsim 15-20^{\circ}$ from the poles, the artifacts are very pronounced in the most anisotropic model, b18a. In this case they have apparently also interacted with the physical instability discussed in Sect. 4.2, affecting a cone as large as $\sim 45^{\circ}$ around the south pole. We feel that the occurrence of a possibly spurious solution over such a large fraction of the computational domain makes this model unsuitable for reasonable analysis, and we will thus skip it from the further discussion. Hence, we will not follow the late-time evolution of this strongly one-sided explosion (resulting from a clearly dominant $l=1$ mode) in this paper, but will constrain ourselves to explosions in which the $l=2$ mode is dominant, and the $l=1$ mode yields only a smaller contribution. We will also try to exclude the regions of these latter models which are affected by the "jets" from all our analyses of expansion velocities and the extent of mixing of different elements, by skipping $15^{\circ}$ of the computational wedge closest to the north and south poles from the data evaluation.

For similar reasons we will also not attempt to calculate the late-time evolution of rotating models in this paper. It is actually true that in a rotating model the polar axis, i.e. the axis of rotation, represents a distinguished direction of the physical system, which is impenetrable for the flow if the specific angular momentum of fluid elements is conserved. In this case the hydrodynamic solution may in fact yield the formation of jetlike polar outflows which are physical. In 2D (i.e. axisymmetric) calculations, employing standard spherical coordinates, it is, however, impossible to study the formation of such features reliably, since the enforced conservation of the z-component of the specific angular momentum leads to numerical artifacts near the poles which will always contaminate the numerical solution.

Unfortunately, a reliable modelling of late-time supernova hydrodynamic instabilities (beyond times of $\sim 10 \mathrm{~s}$ ) for explosions with extreme initial anisotropies and/or rotation appears to require high-resolution 3D simulations of the entire sphere, either in Cartesian coordinates, or with a composite spherical mesh, as e.g. the "cubed sphere grid" described in Ronchi et al. (1996). Both approaches are expected to result in computational tasks that are between two and three orders of magnitude more expensive than the present calculations.

\section{Results}

\subsection{Energetics, timescales, and morphology}

Table 2 provides an overview of all models that we have computed for the $15 M_{\odot}$ blue supergiant progenitor of Woosley et al. (1988) to date. Model b18a (which we will not discuss further due to the reasons mentioned in Sect. 3.2) and model b18b are "medium core luminosity" models with an explosion energy of $10^{51} \mathrm{erg}$. They differ only in the random seed perturbation that we added to the velocity field of Bruenn's post-bounce model to trigger the onset of neutrino-driven convection. They differ hardly in their global parameters like the explosion energy, but show large differences in the morphology, indicating the extremely nonlinear character of the growth of non-radial instabilities in the SN core. Model b23a, on the other hand, is a "high core luminosity", high-energy explosion of $2 \times 10^{51} \mathrm{erg}$. We recall once more that in all of these simulations the core luminosities were held constant for the first second after core bounce (see Sect. 3.1).

In contrast, model T310a (which has been described in detail in Paper I) employed core luminosities that declined exponentially with time. We will include it in the following discussion in order to demonstrate that our new calculations show dramatic quantitative and qualitative differences compared to this older simulation. We strongly encourage the reader to compare the density and velocity plots of model T310a that we presented in Paper I, with the plots for model b23a (Figs. 2, 3 and 8) and model b18b (Figs. 4 and 9) that we show in this paper. The different morphology of the neutrino heated ejecta and the shock within the first few seconds after core bounce is striking. As already stated in the introduction, this is basically a consequence of different explosion timescales and the thus different growth of non-radial instabilities during the shock revival phase by neutrino heating, and the onset of the explosion.

With a timescale $t_{\exp }$ of only $62 \mathrm{~ms}$, model T310a exploded fast - nearly as fast as the typical turnover time of a convective eddy. This neither allowed convection nor the advectiveacoustic shock instability to grow appreciably. Only small convective blobs were formed in the neutrino-heated layer out of the imposed random perturbations, and the shock wave remained undistorted (i.e. spherical). Moreover the flow pattern was dominated by a high-order mode, with about a dozen eddies.

In contrast, models b23a and b18b exploded more slowly by a factor of between two and three (Table 2). Hydrodynamic instabilities in the post-shock flow thus had more time to grow. As in the simulations of Scheck et al. $(2004,2006)$ the initially small blobs in the neutrino-heated layers merged, and ultimately formed a low-order, $l=2$ dominated mode with a smaller $l=1$ mode contribution, exhibiting only two large buoyant bubbles which are separated by a single accretion funnel. In the process, they deformed the shock wave to a prolate, almost peanut-like shape (see Figs. 1b, 1c, 2a and 4a).

The longer explosion time scales also led to larger neutron star masses in models b23a and b18b around one second after core bounce than in model T310a (Table 2). While these masses are still rather low, the comparison shows that the exact value is fairly sensitive to the treatment of the neutrino physics and the assumed boundary conditions (i.e. the neutrino properties and the contraction behaviour of the neutron star core). When the explosion sets in later, the neutron star mass is higher. Moreover it also depends on the structure of the progenitor star (see Scheck et al. 2006 for the corresponding significant variations in case of different $15 M_{\odot}$ progenitors), and can increase due to later 

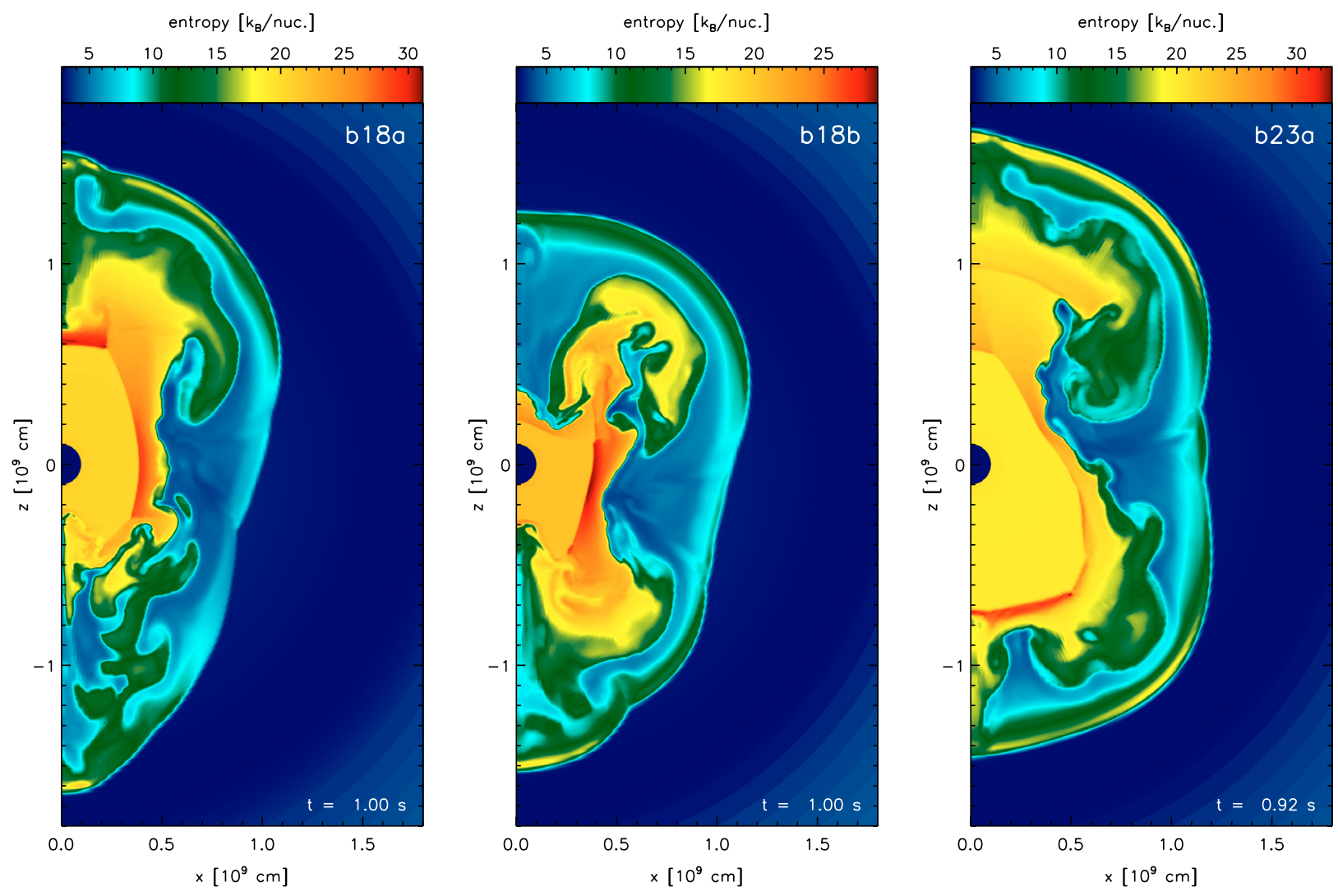

density $\left[\mathrm{g} / \mathrm{cm}^{3}\right]$
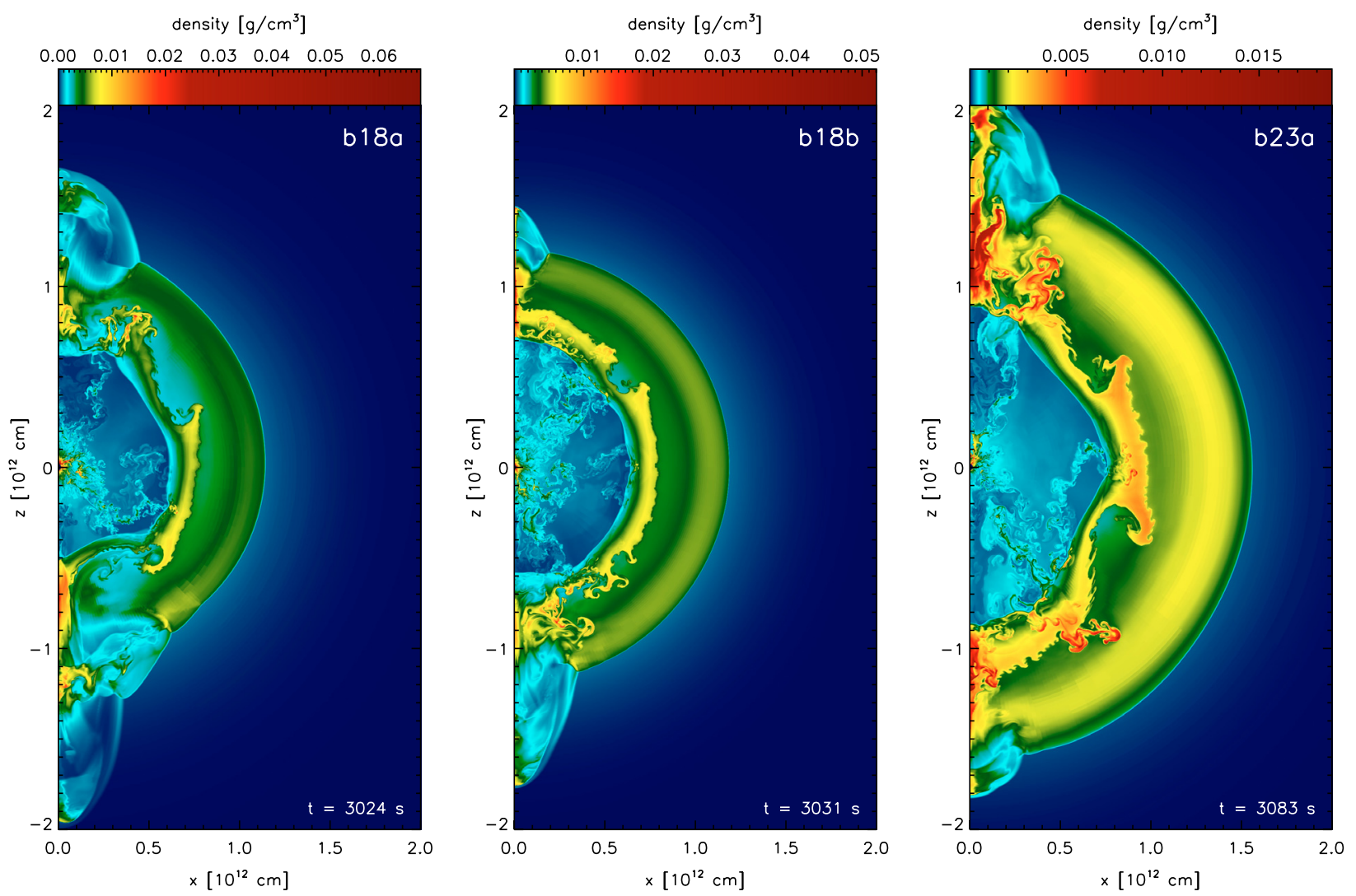

Fig. 1. Top row: entropy distribution (in units of $k_{\mathrm{B}}$ /nucleon) for three of the simulations listed in Table 2 at early times. From left to right a) model b18a at $t=1 \mathrm{~s}, \mathbf{b})$ model $\mathrm{b} 18 \mathrm{~b}$ at $t=1 \mathrm{~s}$, and c) model b23a at $t=0.92 \mathrm{~s}$. Bottom row: density distribution for the same models at later instants. From left to right: d) model b18a at $t=3024 \mathrm{~s}$, e) model b18b at $t=3031 \mathrm{~s}$, and f) model b23a at $t=3083 \mathrm{~s}$. Note the change of shape of the supernova shock (the outermost discontinuity in all plots). Note also the polar jets which deform the otherwise spherical main shock at late times. They are a consequence of our use of a spherical coordinate grid and our assumption of axisymmetry. 

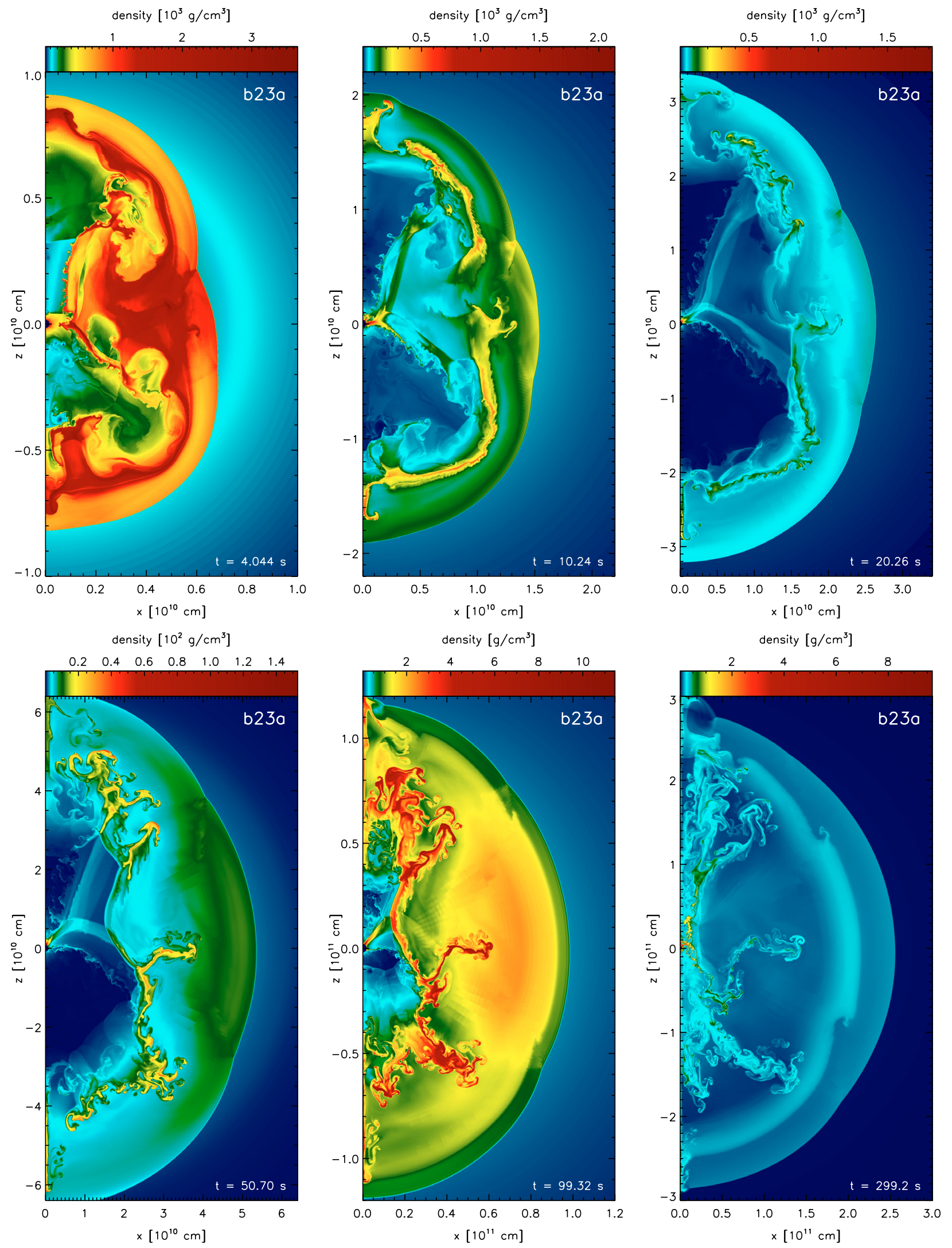

Fig. 2. Snapshots of the density distribution of model b23a for several times. From top left to bottom right a) $t=4 \mathrm{~s}$, b) $t=10 \mathrm{~s}$, c) $t=20 \mathrm{~s}$, d) $t=50 \mathrm{~s}$, e) $t=99 \mathrm{~s}$, and f) $t=299 \mathrm{~s}$. Note the change of the radial scale. The supernova shock is the outermost discontinuity which becomes progressively spherical with time. Note also the growth and outward propagation of the Rayleigh-Taylor mushrooms from the former $\mathrm{Si} / \mathrm{O}$ and $\mathrm{O} / \mathrm{He}$ interfaces of the star, and the onset of the Richtmyer-Meshkov instability at the $\mathrm{He} / \mathrm{H}$ interface (the deformed discontinuity just behind the supernova shock in the plots for $t=99 \mathrm{~s}$ and $t=299 \mathrm{~s}$ ). 

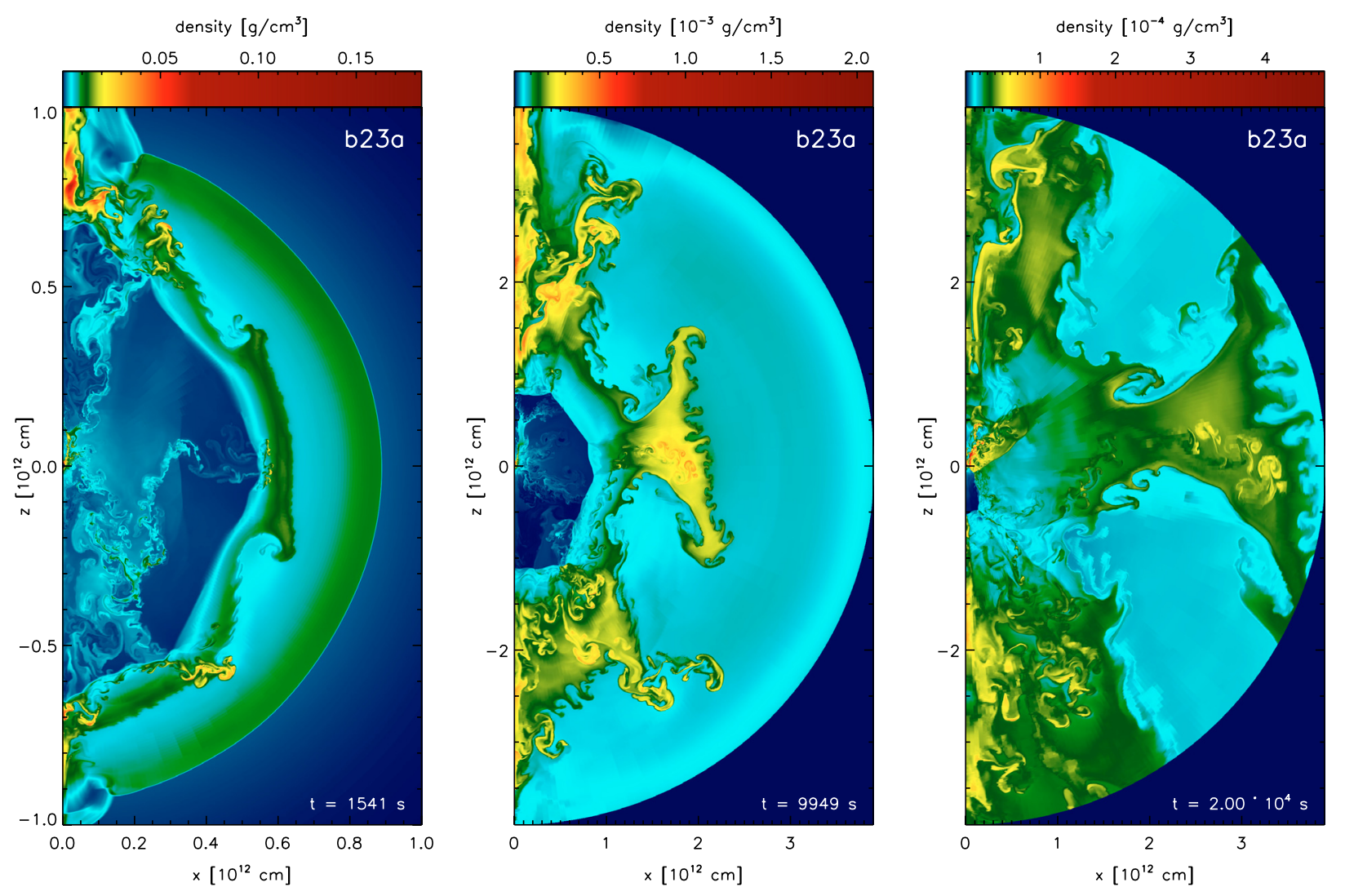

Fig. 3. Same as Fig. 2, but for selected late instants in time. From left to right a) $t \approx 1500 \mathrm{~s}, \mathbf{b}) t \approx 10000 \mathrm{~s}$, and c) $t \approx 20000 \mathrm{~s}$. The outer circular boundary in the last two plots is the outer boundary of the computational domain, which the supernova shock has already left at those times. The shock is only visible in the plot for $t \approx 1500 \mathrm{~s}$ and has become almost completely spherical at this time, while it is decelerating in the hydrogen envelope. Below it, the Richtmyer-Meshkov instability at the $\mathrm{He} / \mathrm{H}$ interface has grown, while in still deeper layers a strong reverse shock decelerates the matter of the He core. Note (by comparing Fig. 3a with Fig. 2f) that a few of the Rayleigh-Taylor clumps have actually reached the $\mathrm{He} / \mathrm{H}$ interface before this reverse shock has managed to form, and that thereby they have escaped an interaction with this shock. Note also the huge hydrogen pockets that are created by the Richtmyer-Meshkov instability.

fallback. We have not traced this fallback in the present work, though.

\subsection{Shock expansion and Richtmyer-Meshkov instability}

The early global anisotropy of the shock in the low-mode models is decisive for the further evolution. It leads to qualitatively new effects in the late-time hydrodynamics compared to model T310a, in which the shock remained spherical from the outset, or the simulation presented in Kifonidis et al. (2000), where the shock had been deformed by rising bubbles during the neutrino-heating phase, but had become spherical by the time it emerged from the He core (see Chevalier \& Soker 1989, for a discussion of the underlying mechanism). Note that in our previous simulations such deformations of the shock due to buoyant neutrino-heated bubbles were in all cases local ones, with deviations of the local to the mean shock radius of at most $20 \%$. In contrast, model b23a has a prolate shock with an initial major to minor axis ratio of $\sim 1.5$. A deformation of this magnitude not only makes the definition of a mean shock radius questionable. It is so substantial that it apparently cannot be smoothed out completely by the time the blast arrives at the $\mathrm{He} / \mathrm{H}$ interface of the Woosley et al. progenitor.

In model b23a the original peanut-like shock gives way to a surface with an equatorial bulge, that forms at the "peanuts' waist" a few seconds after core bounce, and progressively attempts to bring the shock into the spherical shape (Fig. 2). Yet, it requires some time to succeed in this, and when the blast has crossed the $\mathrm{He} / \mathrm{H}$ interface around $100 \mathrm{~s}$ after core bounce its surface still shows two kinks. Kinks in the shock surface are usually found above prominent downflows, and our "two-kink feature" originated from the merging of two such downflows.

The kinks in model b23a are located at similar latitudes in the northern and southern hemispheres (see Fig. 2e). In these spots the shock hits the composition interface obliquely. As a result, it deposits a significant amount of vorticity into the interface layer, which triggers strong Richtmyer-Meshkov mixing (Richtmyer 1960; Meshkov 1969), much as in the simple, planar geometry setup considered by Hawley \& Zabusky (1989) (in fact, our Fig. 3a shows all features that are visible in the plots of their flows, in particular of the "fast-slow" setup that they studied). Two prominent vortices ${ }^{2}$ form, which transport hydrogen-rich gas in the form of two large "pockets" into the helium and metal core. At the same time material from these core layers is dragged outward into the hydrogen envelope, while a vortex sheet due to the Kelvin-Helmholtz instability forms along a significant part of the interface (Figs. 3a-c).

Note that the $\mathrm{He} / \mathrm{H}$ interface is actually susceptible to both, the Richtmyer-Meshkov, and the Rayleigh-Taylor instability, which is due to pressure and density gradients of opposite sign in

\footnotetext{
${ }^{2}$ In the context of axisymmetric simulations all flow features are actually toroidal structures, and the "vortices" are actually "vortex rings".
} 

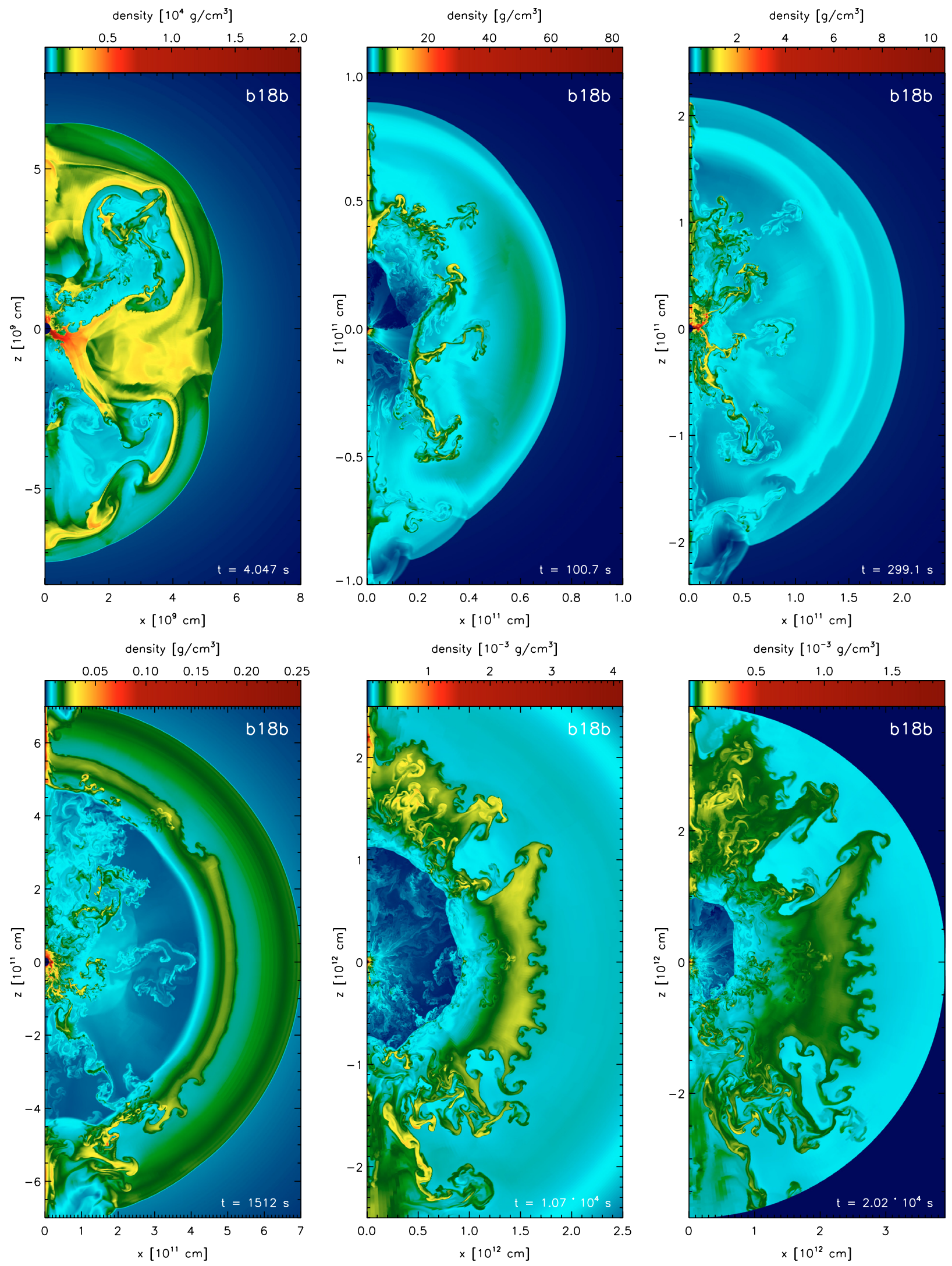

Fig. 4. Snapshots of the density distribution of model b18b for several times. From top left to bottom right a) $t=4 \mathrm{~s}, \mathbf{b}) t=100 \mathrm{~s}$, c) $t=299 \mathrm{~s}$, d) $t=1512 \mathrm{~s}, \mathbf{e}) t \approx 10000 \mathrm{~s}$, and f) $t \approx 20000 \mathrm{~s}$. Note the change of the radial scale. The supernova shock is visible in plots a)-d) only. The outer circular boundary in plot $\mathbf{f}$ ) is again the outer boundary of the computational domain. Note also the more pronounced hemispheric asymmetry of the ejecta at late times as compared to model b23a shown in Fig. 3. 


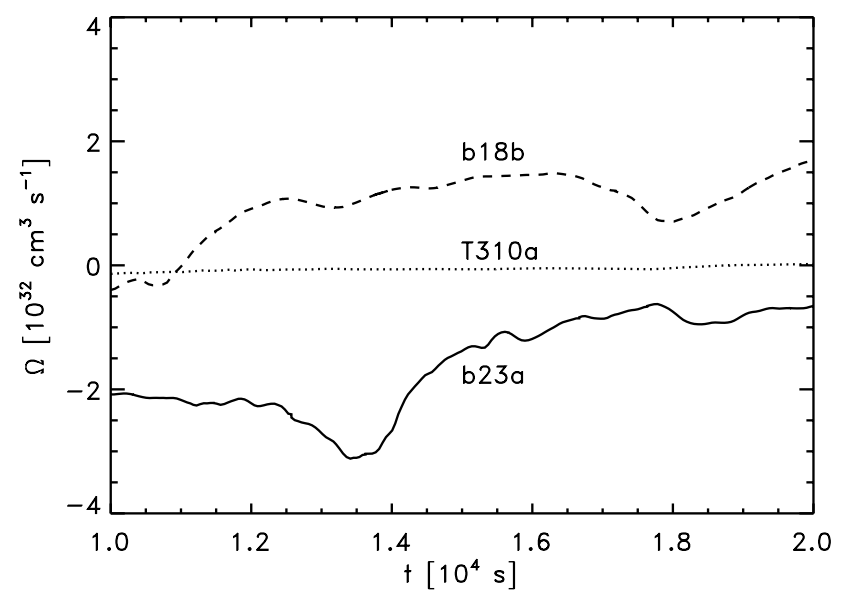

Fig. 5. Integrated vorticity $\Omega$ according to Eq. (4) as a function of time during the late evolutionary phases of model T310a (dotted line), model b23a (solid line), and model b18b (dashed line). Note that $\Omega$ is essentially zero for the high-mode model T310a, while it is of the order $10^{32} \mathrm{~cm}^{3} \mathrm{~s}^{-1}$ for the low-mode models $\mathrm{b} 23 \mathrm{a}$ and $\mathrm{b} 18 \mathrm{~b}$, which experience a strong Richtmyer-Meshkov instability at the $\mathrm{He} / \mathrm{H}$ interface.

this region (see Paper I). At this interface, however, RichtmyerMeshkov instabilities grow faster and appear earlier than RT mushrooms ${ }^{3}$ (compare Figs. $3 \mathrm{~b}$ and $3 \mathrm{c}$ ). On the long run the mixing in fact is a result of both instabilities interacting with each other. Yet, we will still refer to the mixing at the $\mathrm{He} / \mathrm{H}$ interface (a bit loosely) as "Richtmyer-Meshkov instability" in order to make clear that it is the deposition of vorticity by the aspherical shock which is crucial. To demonstrate this, we consider

$\boldsymbol{\Omega}=\int_{V}(\nabla \times \boldsymbol{u}) \mathrm{d} V$,

the integral of the vorticity over the computational domain. In Eq. (3) $\boldsymbol{u}$ is the flow velocity, $V$ is the computational volume, and $\mathrm{d} V$ is the volume element. For 2D axisymmetric calculations in spherical coordinates there is only one component of $\nabla \times \boldsymbol{u}$ that does not vanish, namely $(\nabla \times \boldsymbol{u})_{\varphi}$, and therefore $\boldsymbol{\Omega}$ reduces to the scalar

$\Omega=2 \pi \int_{R_{\mathrm{i}}}^{R_{\mathrm{o}}} \int_{0}^{\pi} \frac{1}{r}\left[\frac{\partial}{\partial r}(r v)-\frac{\partial u}{\partial \vartheta}\right] r^{2} \sin \vartheta \mathrm{d} r \mathrm{~d} \vartheta$,

where $u$ and $v$ are the velocity components in $r$ and $\vartheta$ direction, and $R_{\mathrm{i}}$ and $R_{\mathrm{o}}$ are the inner and outer (open) radial boundaries, respectively. Note that $\Omega$ is a suitable measure for the strength of vortices residing in the domain.

Figure 5 shows the temporal evolution of $\Omega$ for models b23a, b18b, and T310a, and times between $10^{4}$ and $2 \times 10^{4} \mathrm{~s}$. When the shock has already left the grid (which is the case at these times), no contamination of $\Omega$ due to the axial artifacts visible in Fig. 1 is present any more, and the only significant contribution stems from the vorticity deposited early on by the shock at the $\mathrm{He} / \mathrm{H}$ interface. This contribution is of the order of a few $10^{32} \mathrm{~cm}^{3} \mathrm{~s}^{-1}$ for models b23a and b18b, while it is essentially zero for model T310a with its spherical shock.

We have already shown in Paper I that model T310a exhibited only a very weak Rayleigh-Taylor instability at the $\mathrm{He} / \mathrm{H}$ interface, which was triggered by perturbations of this interface

3 The Richtmyer-Meshkov instability is an essentially impulsive instability triggered upon shock passage. The RT instability is taking place on a much longer timescale due to the smooth large-scale gradients of pressure and density at the $\mathrm{He} / \mathrm{H}$ interface.
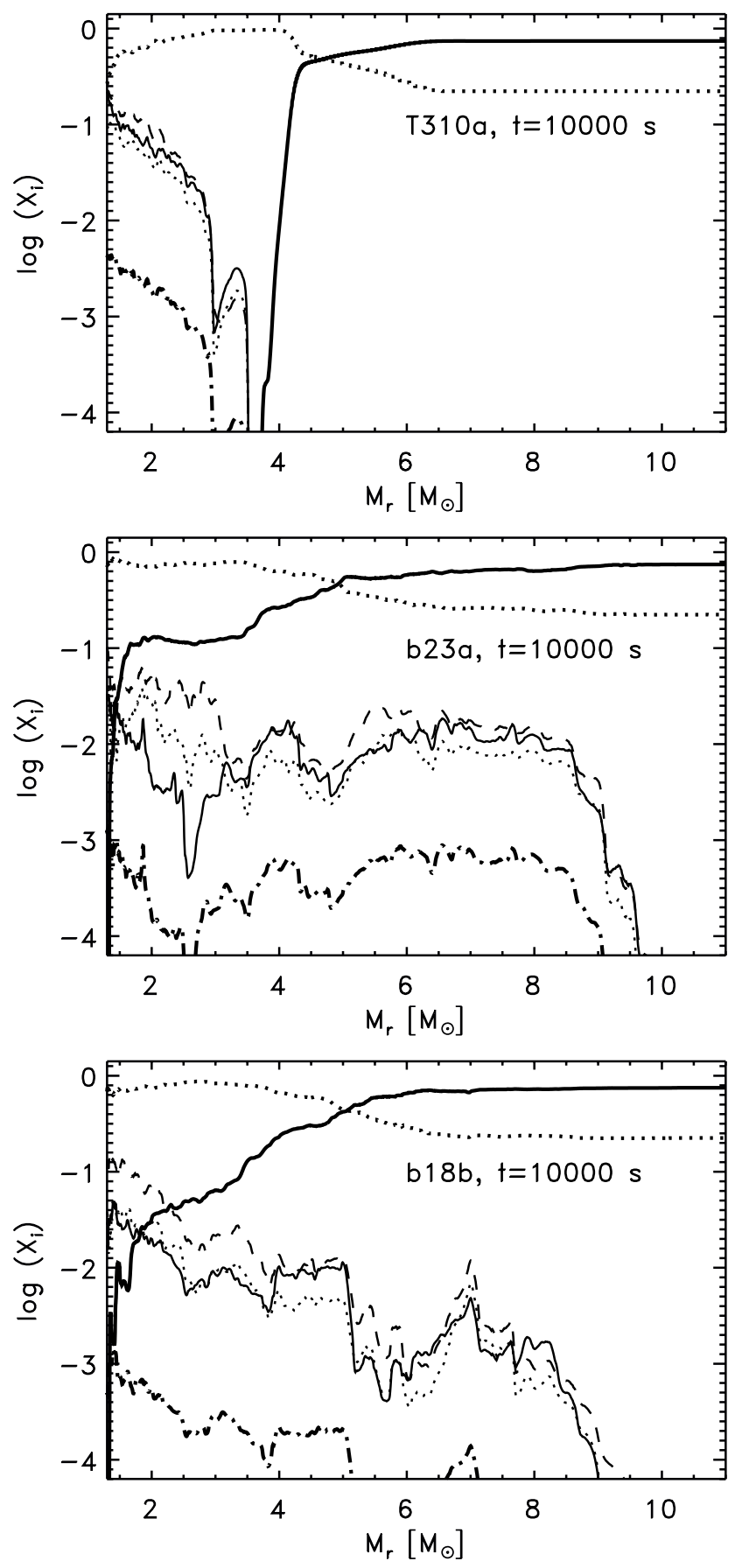

Fig. 6. Extent of the chemical mixing in the simulations at a time of 10000 s. From top to bottom a) model T310a, b) model b23a, and c) model b18b. In all cases the angle-averaged mass fractions of $\mathrm{H}$ (bold solid line), ${ }^{4} \mathrm{He}$ (bold dotted line), ${ }^{16} \mathrm{O}$ (thin dashed line), ${ }^{28} \mathrm{Si}$ (thin dotted line), ${ }^{4} \mathrm{Ti}$ (bold dashed-dotted line), and the iron group (thin solid line), are shown as a function of the enclosed mass. Note the deep inward mixing of hydrogen and the strong outward mixing of the metals in models b23a and b18b, and the lack thereof in model T310a.

due to acoustic noise (i.e. sound waves). Although this led to the growth of a large number of small Rayleigh-Taylor mushrooms, model T310a clearly failed to mix significant amounts of hydrogen into the helium and metal core, i.e. below a mass coordinate of $\sim 4 M_{\odot}$ (compare Fig. 6a). The "high-vorticity" models, $\mathrm{b} 23 \mathrm{a}$ and $\mathrm{b} 18 \mathrm{~b}$, on the other hand, succeed in mixing hydrogen down to a mass coordinate of $\sim 1.3 M_{\odot}$ (Figs. $6 \mathrm{~b}$ and $6 \mathrm{c}$ ), which 

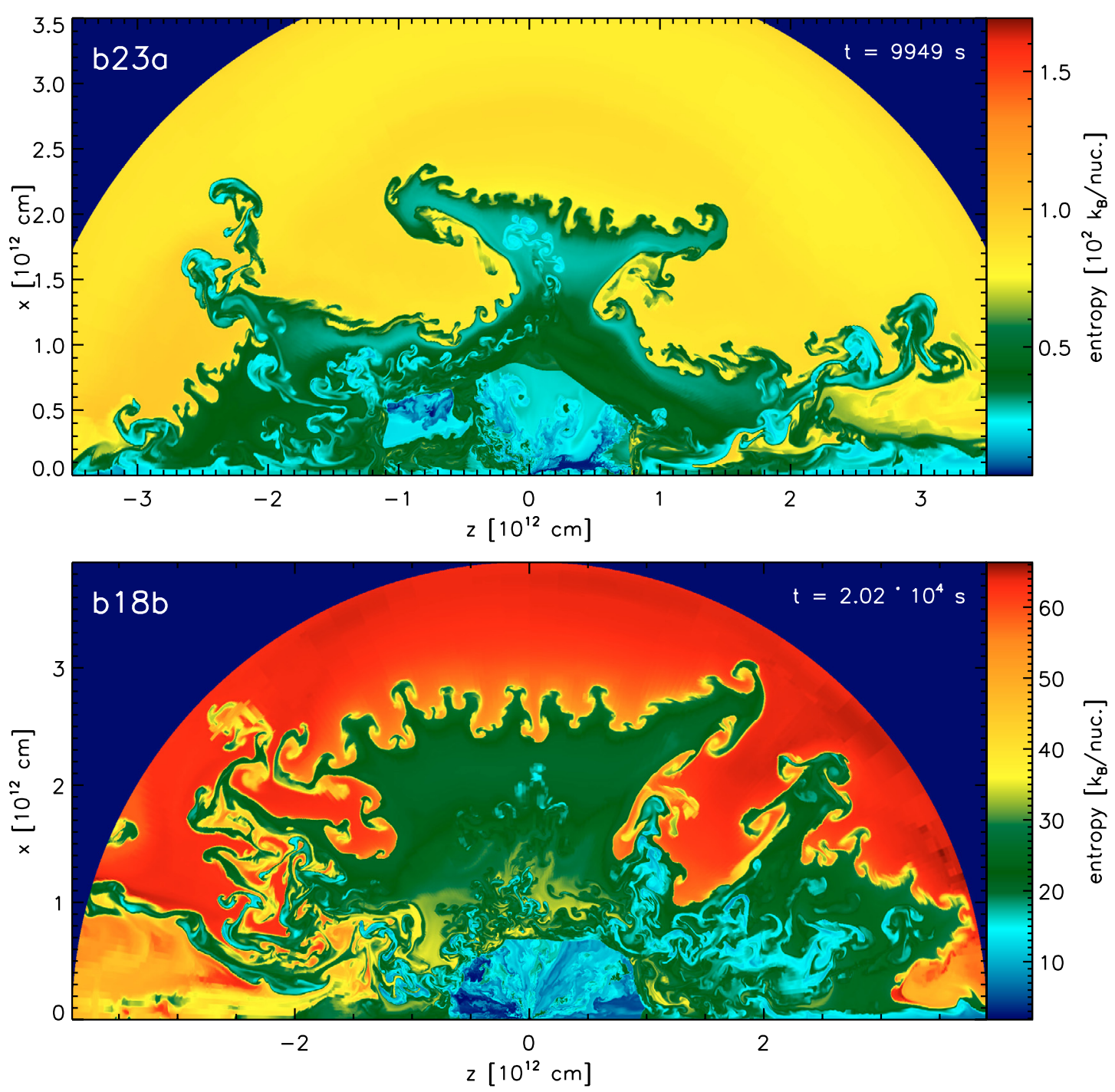

Fig. 7. Entropy distribution (in units of $k_{\mathrm{B}} /$ nucleon) of the low-mode models at late times. From top to bottom: a) model b23a at $t \approx 10000 \mathrm{~s}$ and b) model b18b at $t \approx 20000 \mathrm{~s}$. The color coding has been chosen such that material with different composition can be clearly distinguished. Hydrogen-rich gas is shown in orange or red, while material of the helium core is coded in dark green. Matter from the metal core, which includes nuclei that have been synthesized during the explosion as well as during the hydrostatic evolution of the star, is shown in light and dark blue.

is close to the neutron star mass. This clearly demonstrates that in addition to the short-wavelength Rayleigh-Taylor instability (which can be easily identified in the late evolutionary stages of models b23a and b18b by the typical mushroom-shaped structures, see Figs. 3, 4 and 7), a much more efficient vortical mixing mechanism is present in these models, which operates on much larger scales. Here we identify this vortical mechanism with the Richtmyer-Meshkov instability (see also Hawley \& Zabusky 1989, and the references therein).

A consequence of the nature of this instability is that it leads to a dependence of the final (large-scale) spatial distribution of chemical elements on the initial shape of the shock, i.e. on the spectrum of the superposed unstable modes in the early postshock flow pattern. If, initially, two equally large buoyant highentropy bubbles are present behind the shock, which are separated by a single prominent accretion funnel that is located close to the equatorial plane, i.e. if a dominant $l=2$ mode is present (see Figs. 1c and 2a), then the later distortion of the shock surface still reflects this initial asymmetry of quadrupolar character. In this case two nearly equally large pockets of hydrogen finally develop, which, together with the distribution of the outward dragged material from the helium and metal core, show almost equatorial symmetry (Fig. 7a). If on the other hand, the buoyant bubbles are of different size and the accretion funnel is bent and inclined towards one of the poles, i.e. if there is some admixture of the $l=1$ mode in the initial flow pattern, as in model b18b (see Figs. $1 \mathrm{~b}$ and $4 \mathrm{a}$ ), the $\mathrm{H}$ pockets tend to grow to different sizes. The final hydrogen distribution then develops a pronounced hemispheric asymmetry, as do also the spatial distributions of helium and the metals (Fig. 7b).

Both the deep inward mixing of hydrogen, and the natural development of large-scale, hemispheric asymmetries in the spatial distribution of different elements, have important consequences for the interpretation of observational data of supernova explosions and supernova remnants. The former is required to obtain good fits in light curve modelling, while the latter is probably the cause of the asymmetric iron (and nickel) lines of SN 1987 A. We will return to these issues in Sect. 5.

It is quite astonishing that (unlike the RT instability) the importance of the Richtmyer-Meshkov instability for the 

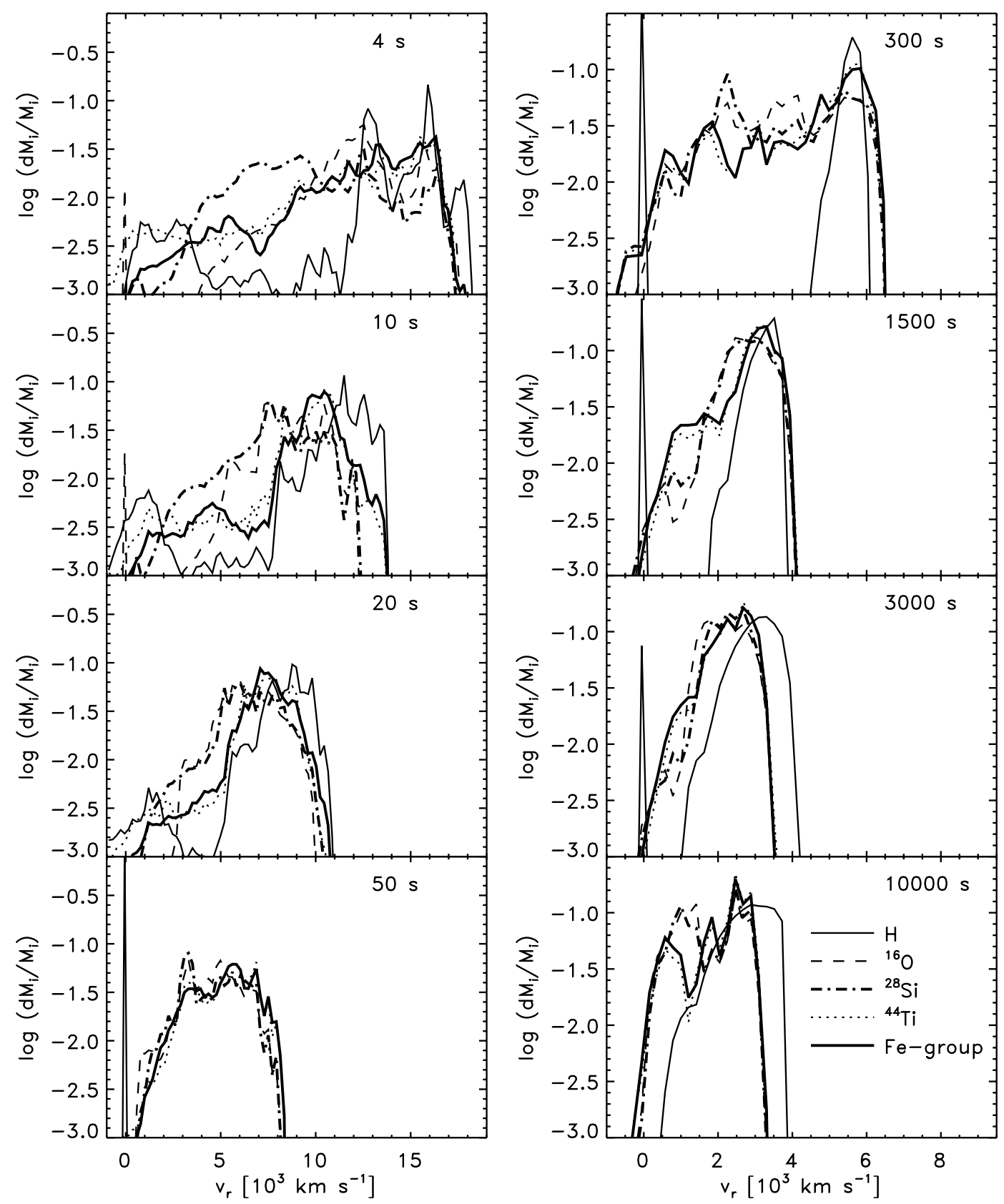

Fig. 8. Logarithm of the fractional mass of different elements as a function of the radial velocity $v_{\mathrm{r}}$ for model b23a at various epochs. Note the different scales for $v_{\mathrm{r}}$ in the left and right panels. Note also that the fractional mass was computed with respect to the mass of the element, $M_{i}$, that is contained on the current extent of the computational grid. For the lighter nuclei $\mathrm{H}$ and ${ }^{16} \mathrm{O}$, this mass is not necessarily identical to the total element mass, because initially our adaptive mesh does not yet cover the outermost stellar layers, and because in the late phases the shocked gas is allowed to stream off the grid. The latter fact is the reason why the hydrogen velocity distribution at $t=10000 \mathrm{~s}$ does not extend beyond $\sim 4000 \mathrm{~km} \mathrm{~s}^{-1}$ (because faster expanding hydrogen has left the grid already).

observational appearance of core collapse supernova explosions has not been widely recognized in previous modelling (but see e.g. Kane et al. 2000, for an exception). This may be, at least in part, due to the fact that in previous studies of aspherical shock propagation through exploding stars this instability was not noticed. Judging from the published information, it is for instance not visible in the calculations of Hungerford et al. (2003, 2005). These authors started their 3D simulations at a time of $100 \mathrm{~s}$ after core bounce from a 1D calculation of the earlier phases of the explosion. Thus the shock had already crossed the $\mathrm{He} / \mathrm{H}$ interface at the time they added asymmetries to the velocity field of their spherically symmetric explosion models. This obviously gave the instability no chance to develop.

In the works of Nagataki (2000) and Yamada \& Sato (1991), on the other hand, who initiated their 2D simulations from aspherical shocks at much earlier times, it was probably the coarse angular resolution of only 100 zones which prevented them from discovering the instability (in the latter paper a diffusive advection scheme with only first-order accuracy in space was employed, too). We think that this demonstrates the importance of 


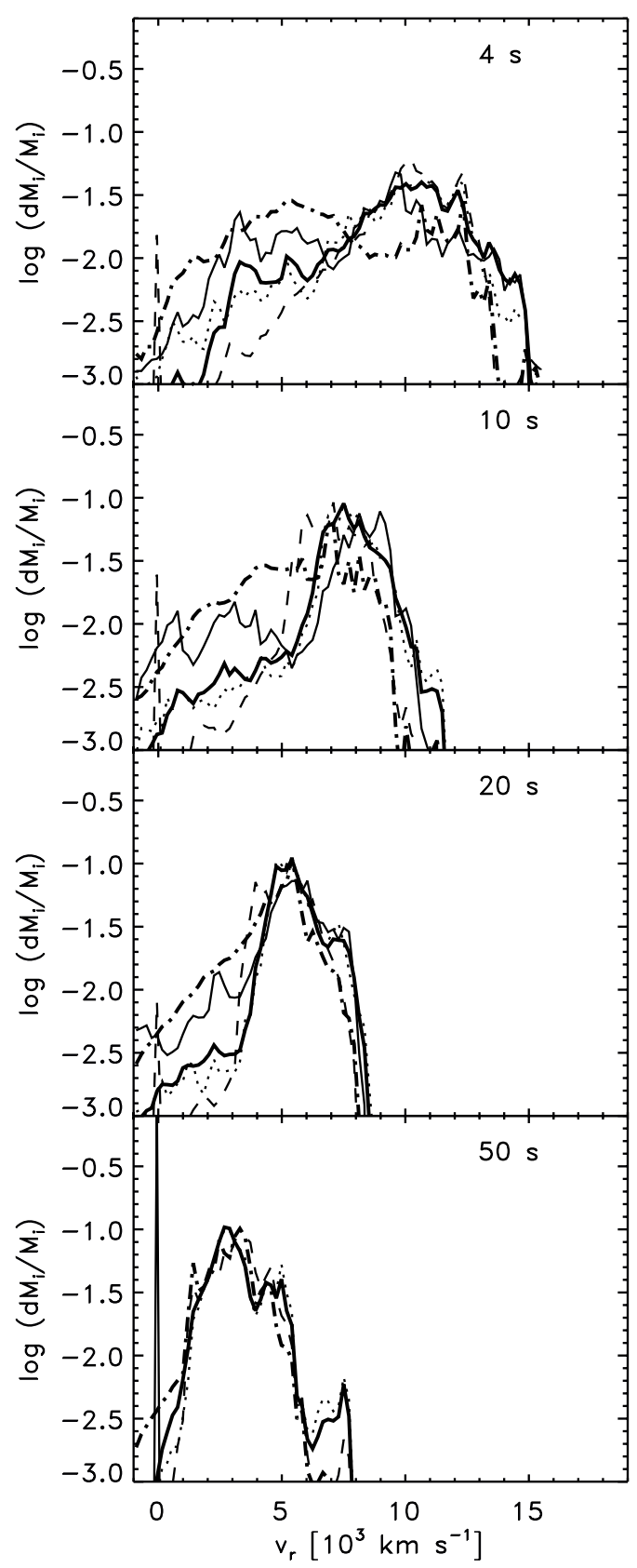

Fig. 9. Same as Fig. 8, but for model b18b.

two points which we have already addressed in Paper I, namely that modelling the mixing in core-collapse $\mathrm{SNe}$ requires one to follow the evolution from the earliest phases of the explosion in more than one dimension (in order to avoid biasing the late-time evolution). Furthermore, high resolution and an excellent advection scheme are essential for resolving and properly describing the relevant fluid instabilities.

\subsection{Metal clumps and Rayleigh-Taylor instability}

The unstable modes that become dominant within the first second of the explosion, do not only determine the morphology of the shock, and thereby the sequence of events at the $\mathrm{He} / \mathrm{H}$ interface. They also provide the perturbations for the Rayleigh-Taylor unstable $\mathrm{Si} / \mathrm{O}$ and $\mathrm{O} / \mathrm{He}$ interfaces of the progenitor. We have

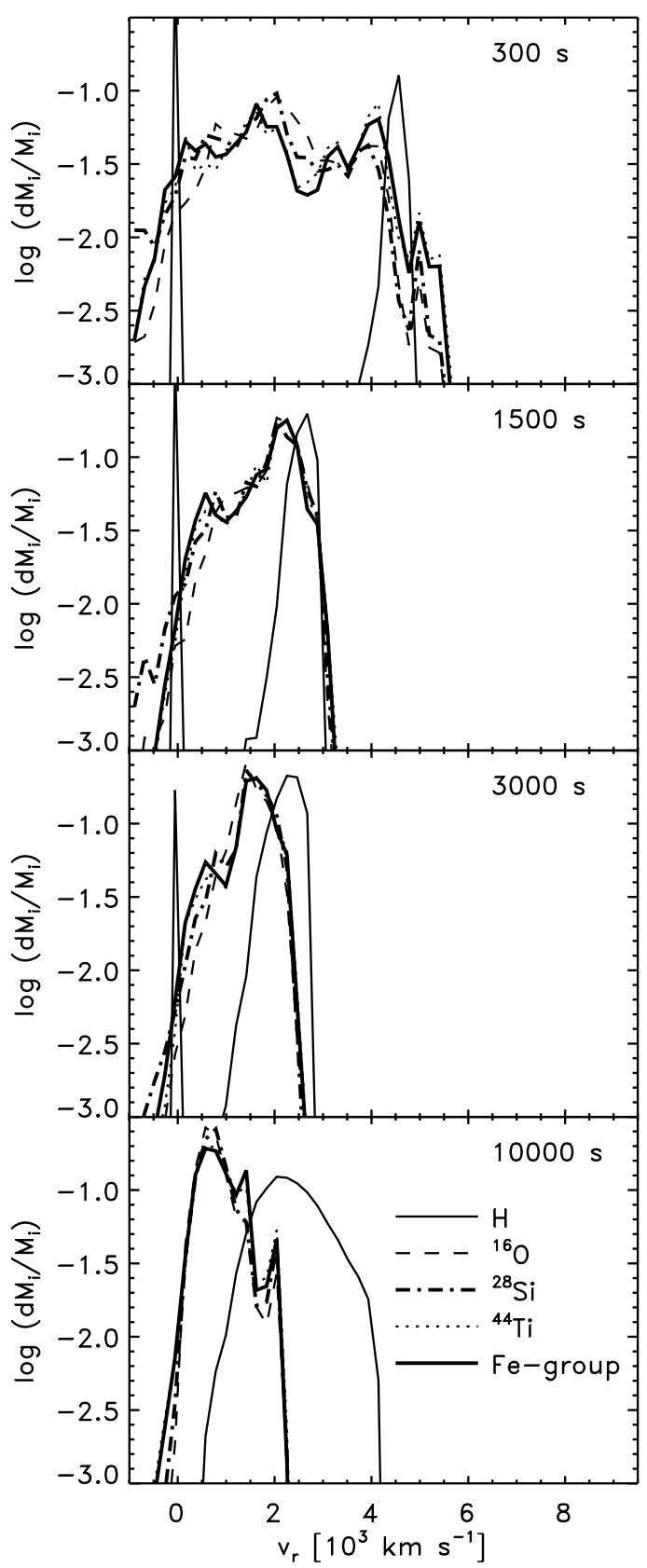

noticed in Paper I that the wavelength of these perturbations determines the number of Rayleigh-Taylor fingers that grow at these interfaces, and that it characterizes the flow pattern until late times. This is also supported by the recent work of Miles (2004), who has developed a Rayleigh-Taylor bubble merger model for compressible flows. He concluded that unless the shock Mach number and the mode number of the initial perturbation are very high, the system will not loose memory of the original perturbation spectrum.

This is indeed the case in all models that we have computed to date. Model T310a developed about a dozen Rayleigh-Taylor fingers out of a comparable number of flow features that formed during the phase of neutrino-driven convection near the $\mathrm{Si} / \mathrm{O}$ and $\mathrm{O} / \mathrm{He}$ interfaces (Paper I). The low-mode models b23a and $\mathrm{b} 18 \mathrm{~b}$, on the other hand, lead to the growth of only about three to four, rather large, Rayleigh-Taylor mushrooms (Figs. 2 and 4). In 
what follows we will keep referring to the case of model T310a as a high-order, and to this of models b23a and b18b as a loworder mode, in order to distinguish them clearly. In the sense of Miles (2004) all of our models bear "low-mode" perturbations, because they all satisfy his condition for retainment of the initial mode number.

Besides having a different number and size of Rayleigh-Taylor "clumps", both cases also differ strongly in the velocity distributions of nucleosynthetic products. The low-mode models show much broader distributions in velocity space for iron-group nuclei and for the isotopes ${ }^{16} \mathrm{O},{ }^{28} \mathrm{Si}$ and ${ }^{44} \mathrm{Ti}$ a few seconds after bounce than model T310a (compare the plots for $t=4 \mathrm{~s}$ in Figs. 8 and 9 with Fig. 16 of Paper I).

Even more importantly, the maximum velocities of these nuclei are significantly larger than in the high-mode model T310a. In model b23a we find maximum metal velocities $4 \mathrm{~s}$ after bounce that are about $40 \%$ larger than in model T310a. But even in the low-energy, low-mode model b18b, whose explosion energy is nearly half that of model T310a (1.0 vs. $1.7 \times 10^{51} \mathrm{erg}$, respectively), the corresponding velocities are by about $20 \%$ larger.

The spatial distribution of kinetic energy in the low and highmode cases is fundamentally different. In model T310a most of the kinetic energy was contained in an essentially spherical shell behind the shock (similar to a 1D simulation), and there was only little kinetic energy in the two-dimensionally perturbed region containing the bubbles. The weak neutrino-driven convection in model T310a was not able to boost the velocities inside those bubbles relative to the mean expansion velocity of the background flow. In the low-mode models this is different. The high-entropy bubbles and the higher-density pockets enclosed by them contain most of the kinetic energy of the explosion.

The higher metal velocities during the first few seconds after core bounce lead to significant differences in the late-time evolution of the low-mode models as compared to model T310a. The metal clumps now propagate faster through the He core of the star, keeping closer contact to the outward sweeping supernova shock. In both, model b23a and model b18b, the fastest clumps have almost reached the $\mathrm{He} / \mathrm{H}$ interface around $300 \mathrm{~s}$ after core bounce (see Figs. $2 \mathrm{f}$ and $4 \mathrm{c}$ ). At this time the strong reverse shock that will develop below this interface due to the deceleration of the main shock in the hydrogen envelope (compare Fig. 3a and Paper I), has not yet formed. Hence, an interaction of the fastest clumps with this reverse shock, which leads to the dramatic slow-down of the metals beyond a time of $1500 \mathrm{~s}$ in model T310a (Paper I), does not happen in the low-mode models!

Instead, the fastest clumps encounter a layer in which the density gradient, though steepening, is still relatively flat. Their motion relative to the background flow thus never becomes supersonic, and deceleration enhanced by supersonic drag is absent. Hence the maximum velocities of iron-group nuclei and other elements decrease only slightly at late times, e.g. from $3500 \mathrm{~km} \mathrm{~s}^{-1}$ at $t=1500 \mathrm{~s}$ to $3300 \mathrm{~km} \mathrm{~s}^{-1}$ at $t=10000 \mathrm{~s}$ in model b23a (Fig. 8). These velocities remain unchanged until the time when the clumps start to leave the outer boundary of our grid, which happens at $t \approx 12000 \mathrm{~s}$ in model b23a.

Strong reduction of a late-time deceleration may also be favoured by the fact that the metal clumps become engulfed in the Richtmyer-Meshkov instability at the $\mathrm{He} / \mathrm{H}$ interface. The vortical energy deposited early on in these layers by the shock, represents a reservoir of kinetic energy that can possibly be tapped during the late-time hydrodynamic evolution, and would have been unavailable to the clumps if the shock had been spherically symmetric when it crossed the interface (as it was the case in model T310a).

Summarizing these findings, we can state that by following the late-time hydrodynamic evolution of neutrino-driven explosions which were dominated by early, low-mode hydrodynamic instabilities, we have for the first time obtained a consistent (in the sense defined in Sect. 1) solution to the nagging "nickel discrepancy" problem (Herant \& Benz 1992), i.e. we have obtained maximum velocities for the bulk of the metal-rich material (in model b23a) which are in good agreement with those of SN 1987 A. This result calls for a more careful inspection of the simulations in order to judge whether they also fulfill other observational constraints obtained from this supernova.

\section{Comparison with SN 1987 A}

The most satisfactory approach for verifying whether our simulations are consistent with the observational data of SN 1987 A would, of course, be the computation of light curves, spectra and the degree of polarization from the $2 \mathrm{D}$ hydrodynamic models. This is beyond the scope of the present paper and will be attempted in future work.

Here we will confine ourselves to a semi-quantitative comparison with a check list that we assembled from the results of previous observational and (one-dimensional) modelling work. This list, which any successful model of SN 1987 A needs to pass, consists of the following items:

1. The high velocities of iron-group nuclei, or, given that ultimately homologous expansion establishes, the outward mixing of these nuclei in mass. We will focus here on the mixing in velocity space since this could be measured directly by observations of the $\mathrm{Fe}$ and $\mathrm{Ni}$ infrared lines. The mixing in the mass coordinate can be deduced only indirectly, on the basis of theoretical models, and may thus be less reliable.

Haas et al. (1990) and Colgan et al. (1994) have carried out observations of the [Fe II] $18 \mu \mathrm{m}$, [Fe II] $26 \mu \mathrm{m}$, and [Ni I] $6.6 \mu \mathrm{m}$ lines at 410 and 640 days after the explosion. All of these lines show broad wings. In addition, there is an isolated high-velocity emission feature in the red wing of both the iron lines at day 410, and the nickel line at day 640, which indicates that a single clump with $\sim 3 \%$ of the total iron mass moves away from the observer with a velocity of $3900 \mathrm{~km} \mathrm{~s}^{-1}$ (Haas et al. 1990). Jennings et al. (1993) give an expansion velocity of $3150 \mathrm{~km} \mathrm{~s}^{-1}$ for this high-velocity feature, based on independent observations of the [Fe II] $18 \mu \mathrm{m}$ line at day 613. The [Ni I] $6.6 \mu \mathrm{m}$ measurements of Colgan et al. (1994) at day 640 exhibited probably the highest signalto-noise ratio but were affected by absorption due to dust, which had formed in the ejecta between days 410 and 640 . Colgan et al. (1994) thus regard the [Fe II] lines at day 410 as providing the best evidence for the amount of iron-group elements at high velocities. From the width of these lines Haas et al. (1990) concluded that $\gtrsim 4 \%$ of the total iron (excluding the high-velocity feature) has an expansion velocity $\gtrsim 3000 \mathrm{~km} \mathrm{~s}^{-1}$.

Early nickel mixing with significantly higher velocities (above $5000 \mathrm{~km} \mathrm{~s}^{-1}$ ) has been postulated by Mitchell et al. (2001) in order to get sufficient excitation for the hydrogen Balmer lines in their steady state spectral models for day 4. Utrobin \& Chugai (2005), however, have recently shown that such high (bulk) nickel velocities are not required to explain the observations during the photospheric phase, if one takes into account the effects of time-dependent ionization in 
modelling the spectra. With their time-dependent ionization code excellent fits to the $\mathrm{H} \alpha$ profiles are obtained if the ${ }^{56} \mathrm{Ni}$ is mixed out to only $\sim 3000 \mathrm{~km} \mathrm{~s}^{-1}$. Further work which provides compelling evidence of such more moderate velocities for the bulk of the ${ }^{56} \mathrm{Ni}$ (and, for instance, includes modelling of the X-ray and $\gamma$-ray emission of SN 1987 A) is summarized in the review of McCray (1993). Some more recent papers are the light curve study of Utrobin (2004), and the work of Fassia \& Meikle (1999). The latter authors have applied spectral synthesis models to the He I $10830 \AA$ Aine after day 10 and found that about $3 \%$ of the total ${ }^{56} \mathrm{Ni}$ mass must be mixed to above $3000 \mathrm{~km} \mathrm{~s}^{-1}$, but that the ${ }^{56} \mathrm{Ni}$ concentration must be negligible above $3900 \mathrm{~km} \mathrm{~s}^{-1}$. They also demonstrated that models with this amount of mixing are able to reproduce the $\gamma$-ray light curves and the late time infrared line velocities.

2. The marked asymmetry of the [Fe II] and [Ni I] infrared lines (Haas et al. 1990; Spyromilio et al. 1990; Jennings et al. 1993; Colgan et al. 1994). Besides the (localized) "highvelocity-feature" that we already mentioned above, these lines also show a global red/blue asymmetry. Its origin was probably anisotropic expansion of the ejecta on a global scale (Haas et al. 1990; Jennings et al. 1993).

3. The total ${ }^{56} \mathrm{Ni}$ mass of $\sim 0.07 M_{\odot}$, which is required to power the light curve (Woosley 1988; Suntzeff \& Bouchet 1990; Bouchet et al. 1991).

4. The very low minimum hydrogen velocities. The mere fact that the $\mathrm{H} \alpha$ line profile observed by Phillips et al. (1990) on day 498 is not flat-topped implies that there is no large hydrogen-free cavity in the slowly expanding central layers of the ejecta (V. Utrobin, private communication). From the narrow core of the $\mathrm{H} \alpha$ line profiles of Phillips et al. (1990) around day 800, Kozma \& Fransson (1998) inferred a minimum hydrogen velocity of $\$ 700 \mathrm{~km} \mathrm{~s}^{-1}$. Mixing of hydrogen into the $\mathrm{SN}$ core, combined with the outward mixing of ${ }^{56} \mathrm{Ni}$, is also necessary to fit the broad peak of the light curve (e.g. Woosley 1988; Shigeyama \& Nomoto 1990; Blinnikov 1999). In the models of Blinnikov (1999) hydrogen is mixed down to a mass coordinate of $\sim 1.3 M_{\odot}$.

5. The prolate deformation of the (inner) ejecta with an axis ratio of roughly $2: 1$ that has been found by high resolution imaging with the HST, and which has been observed indirectly by earlier spectropolarimetry measurements (see Wang et al. 2002, and the references therein). It is particularly noteworthy that the degree of polarization was only $0.1 \%$ two days after the explosion and increased to $1.1 \%$ at about day 200 , indicating that the scattering surface became increasingly asymmetric as the observations probed the deeper layers of the ejecta (Wang et al. 2002).

6. The constraints on the ratio of the explosion energy to ejected mass, $E_{\exp } / M$, that have been deduced from modelling of the light curve and the hydrogen lines. Utrobin $\&$ Chugai (2005) give a value of $0.83 \times 10^{50} \mathrm{erg} M_{\odot}^{-1}$. Blinnikov (1999) finds, from a multigroup radiation hydrodynamic study, $E_{\exp } / M=(0.75 \pm 0.17) \times 10^{50} \mathrm{erg} M_{\odot}^{-1}$ (based on $E_{\exp }=(1.10 \pm 0.25) \times 10^{51} \mathrm{erg}$, and an assumed ejected mass of $\left.14.7 M_{\odot}\right)$, while from the models listed in Table 2 of Shigeyama \& Nomoto (1990) an average value of about $0.76 \times 10^{50}$ erg $M_{\odot}^{-1}$ can be inferred.

We have already noted that in the high-energy model, b23a, the (final) maximum iron-group velocities are about $3300 \mathrm{~km} \mathrm{~s}^{-1}$. Furthermore, a few per cent of the total iron-group mass in this model is expanding with velocities $\gtrsim 3000 \mathrm{~km} \mathrm{~s}^{-1}$ (compare
Fig. 8), as required by the observations of Haas et al. (1990). Our simulations are thus able to reproduce the maximum velocities of the bulk of the iron-group mass in SN 1987 A. The explanation of single, even faster clumps (Utrobin et al. 1995) might require different initial conditions, or higher resolution, or will have to await future 3D simulations. Note also that in our low-energy model, b18b, the highest iron-group velocities are $\sim 2200 \mathrm{~km} \mathrm{~s}^{-1}$, which is too low and indicates that the explosion energy was higher than $10^{51}$ erg (see also the comments with respect to item six below).

To be consistent with the second item of the above list, the simulations are required to exhibit variations of the iron velocities, and/or the iron concentration with respect to the latitudinal angle on a large scale, preferably in the form of a hemispheric asymmetry. In this case one would measure higher velocities, and/or a larger line-flux from one hemisphere of the ejecta than from the other. Our models indeed develop such hemispheric asphericities, if a sufficiently large contribution of the $l=1$ mode to the shock morphology is present after the neutrino-heating phase. This, on the other hand, depends on the imposed initial perturbations, which affect the formation and merging of the neutrino-heated bubbles during the first $\sim 300 \mathrm{~ms}$ of the explosion. Since this merging is a chaotic and highly nonlinear process (cf. Scheck et al. 2006), the development of a hemispheric asymmetry is in the end governed by stochastics. Model b18b shows such a pronounced asymmetry, while in model b23a (which fits most of the other check-list items better than model b18b) such an asymmetry is there, too, but weaker (Fig. 7). Yet, the outcome might have well been reversed if different initial perturbations were used. We refrain from demonstrating this here, as this would require us to calculate a large sample of models.

A reproduction of item three hinges very sensitively on the electron fraction, $Y_{\mathrm{e}}$, of the neutrino-heated ejecta. The local $Y_{\mathrm{e}}$ is in turn a result of the competition of the charged-current reactions

$v_{\mathrm{e}}+\mathrm{n} \leftrightharpoons \mathrm{e}^{-}+\mathrm{p}$

$\bar{v}_{\mathrm{e}}+\mathrm{p} \leftrightharpoons \mathrm{e}^{+}+\mathrm{n}$.

Electron capture on protons and $\bar{v}_{\mathrm{e}}$ absorption by protons decrease $Y_{\mathrm{e}}$ and make the matter more neutron rich. On the other hand, the inverse processes of positron capture on neutrons and $v_{\mathrm{e}}$ absorption by neutrons drive the matter to the protonrich side. The rates for the $v_{\mathrm{e}}$ and $\bar{v}_{\mathrm{e}}$ captures depend sensitively on the local $v_{\mathrm{e}}$ and $\bar{v}_{\mathrm{e}}$ spectra, and hence they require an energy-dependent transport treatment for their accurate calculation. With a gray transport scheme like ours this can be done only approximately, resulting in an uncertainty in $Y_{\mathrm{e}}$ during nuclear freezeout of the order of several per cent. Since a variation of only a few per cent in $Y_{\mathrm{e}}$ can result in a more than $100 \%$ change in the ratio of the ${ }^{56} \mathrm{Ni}$ yield relative to the yields of other (e.g. more neutron rich) iron-group nuclei, it is not possible from the present calculations to determine reliable isotopic yield ratios within the iron-group. We can, however, give an upper limit for the ${ }^{56} \mathrm{Ni}$ yield, obtained from the $Y_{\mathrm{e}}$-insensitive total yield of iron-group nuclei. This upper limit amounts to $0.15 M_{\odot}$ and $0.11 M_{\odot}$ in models b23a and b18b, respectively, and is compatible with the fact that $0.07 M_{\odot}$ of ${ }^{56} \mathrm{Ni}$ were produced in SN $1987 \mathrm{~A}$.

The consistency of the simulations with item four is easily verified from Figs. 8 and 9, which show that the lowest hydrogen velocities in both models b23a and b18b are $\sim 500 \mathrm{~km} \mathrm{~s}^{-1}$. That the models are also consistent with the extent of hydrogen mixing assumed in lightcurve modelling is best checked by 
comparing our Fig. 6 with Fig. 2 of Blinnikov (1999). The hydrogen mass fraction profile used by Blinnikov to obtain a good fit to the observed lightcurve is almost indistinguishable from the ones that we obtain for models b23a and b18b. The sharp drop interior to $\sim 1.5 M_{\odot}$ and even the plateau at $\log X=-1$ for mass coordinates $1.5 M_{\odot}<M_{\mathrm{r}}<3.5 M_{\odot}$ are reproduced excellently.

Consistency with item five can be inspected qualitatively from Figs. 3, 4, and 7, which demonstrate that at late times the inner ejecta (i.e. the helium and metal-rich material) in our simulations exhibit a global anisotropy. The major to minor axis ratio of the isodensity contour corresponding to the $\mathrm{He} / \mathrm{H}$ interface is roughly 1.6. The decrease of the asymmetry of the ejecta with radius inferred from the spectropolarimetry data might be explained from the fact that the shock in our models (baring the numerical artifacts near the poles) becomes spherically symmetric once it has propagated through the inner hydrogen envelope (see the plots for $1500 \mathrm{~s}$ in Figs. 3 and 4). This is an important feature of our models which clearly distinguishes them from earlier simulations, e.g. from the asymmetric models presented in Yamada \& Sato (1991). A more detailed comparison with the spectropolarimetry data - beyond the qualitative level that we attempt here - is difficult because it requires a calculation of the (time-dependent) location of the photosphere in our models, which we did not perform.

This leaves us with item six. For our $15 M_{\odot}$ progenitor and model b23a, with its explosion energy of $2.0 \times 10^{51} \mathrm{erg}$, we obtain an energy-to-mass ratio of $1.4 \times 10^{50} \mathrm{erg} M_{\odot}^{-1}$ (after accounting for a neutron star of $1.2 M_{\odot}$ and ignoring possible fallback). This is a bit on the high side. For the low-energy model b18b we get $0.7 \times 10^{50} \mathrm{erg} M_{\odot}^{-1}$, which is in much better agreement with the lightcurve modelling, but for this model the maximum iron-group velocities are only $\sim 2200 \mathrm{~km} \mathrm{~s}^{-1}$, which is in conflict with item one. Still, we think that with a more careful choice of the progenitor model and of the explosion energy, it will be possible to obtain both, agreement with the observed maximum iron-group velocities, and a closer reproduction of the $E_{\exp } / M$ ratio because

- the mass of the SN 1987 A progenitor may have been in the 18-20 $M_{\odot}$ range (Woosley 1988);

- a lowering of the explosion energy from $2.0 \times 10^{51}$ erg to $1.5 \times$ $10^{51} \mathrm{erg}$ will lower our maximum metal velocities only by a factor of $\sim 1.15$ (because these velocities scale approximately with the square root of the explosion energy);

- in three dimensions it is expected that, for the same explosion energy, larger maximum metal velocities will be obtained (due to the smaller drag experienced by truly threedimensional clumps, see Kane et al. 2000).

In summary, the low-mode models, and in particular the highenergy case b23a, are in at least qualitative agreement with the items listed above. In many cases even an astonishingly good quantitative agreement has been obtained. This is remarkable, especially if one considers the fact that there is only very little room for "fine-tuning" of parameters in the calculations that we have presented here. The freedom we have to adjust the model is limited to the choice of the initial random seed perturbation (which influences the relative contribution of different low-order unstable modes in the post-shock flow during the shock revival phase), and the parametrization of the inner boundary condition which determines the (electron flavour) neutrino flux from the neutron-star core, $L^{\text {core }}$, and thereby the explosion time scale and energy of the simulation (cf. Scheck et al. 2006). Note also that the core neutrino flux is only a part of the total neutrino flux in our calculations. A significant part of the total neutrino luminosity is contributed by the neutrino emission of mass accreted onto the neutron star, which is taken into account by our transport scheme (see Scheck et al. 2006). The consistency of our models with the above list therefore makes us confident that the long-standing mystery concerning the origin of the major observational properties of SN 1987 A may have found its solution.

\section{Conclusions}

The main conclusion of the present study is that if a low-mode hydrodynamic instability (more precisely, a quadrupole, $l=2$, mode-dominated instability with a smaller contribution from a dipole, $l=1$, mode) establishes during the first second of a supernova in a standard $15 M_{\odot}$ blue supergiant star (see Scheck et al. 2004, 2006 for the requirements of this), then the ensuing explosion possesses properties which are in remarkable quantitative agreement with the observational data of SN 1987 A.

Among other features, explosions of this type (with an energy of $\sim 2 \times 10^{51}$ erg for the progenitor model we used) exhibit final iron-group velocities of up to $3300 \mathrm{~km} \mathrm{~s}^{-1}$, strong mixing at the $\mathrm{He} / \mathrm{H}$ composition interface, with hydrogen being mixed downward in velocity space to only $500 \mathrm{~km} \mathrm{~s}^{-1}$, and a final prolate anisotropy of the inner ejecta with a major to minor axis ratio of about 1.6.

The success of low-mode explosions to reproduce these observations is based on two effects: the long-wavelength pattern with which the Rayleigh-Taylor unstable $\mathrm{Si} / \mathrm{O}$ and $\mathrm{O} / \mathrm{He}$ interfaces of the presupernova are perturbed, and the initial global deformation of the shock, which induces a strong RichtmyerMeshkov instability at the $\mathrm{He} / \mathrm{H}$ interface.

Compared to our previous models (Kifonidis et al. 2003), which were dominated by high-mode neutrino-driven convection, we find that the low-mode unstable models presented here show significantly (i.e. up to $40 \%$ ) higher initial metal clump velocities. These high velocities, in turn, keep the timescale of clump propagation through the He core short - in particular shorter than the timescale of reverse shock formation near the $\mathrm{He} / \mathrm{H}$ interface. Hence the fastest clumps in the low-mode models never have to interact with this reverse shock, and are thus not slowed down, in marked contrast to the high-mode case (Kifonidis et al. 2003).

The occurrence of a strong Richtmyer-Meshkov instability at the $\mathrm{He} / \mathrm{H}$ interface is of crucial importance for producing the strong inward mixing of hydrogen and the global anisotropy that is present during the late expansion of the inner ejecta. The early deposition of vorticity into the interface layer by the initially aspherical shock (at $t \approx 100 \mathrm{~s}$ ) is responsible for the fact that a late-time global anisotropy of the inner ejecta can develop (at $t \approx 10000 \mathrm{~s}$ ), although the shock itself has long become spherical by that time.

We find that the large-scale anisotropy imprinted on the ejecta and the early shock by low-mode hydrodynamic instabilities during the shock revival phase, and the ensuing RichtmyerMeshkov instability at the $\mathrm{He} / \mathrm{H}$ interface are all that is needed to reproduce the major observational features of SN 1987 A. In other words, mechanisms like jet formation, anisotropic neutrino emission, and rotation of the progenitor are not required to explain the observational characteristics we have summarized in Sect. 5, provided that due attention is paid to account for all hydrodynamic instabilities that occur in the problem and for their mutual interaction.

A coherent picture of supernova explosions, powered by the neutrino-heating mechanism, thus seems to emerge, in which 
non-radial instabilities of the accretion shock like the advectiveacoustic instability of Foglizzo \& Tagger (2000) and Foglizzo (2002) (see in particular Foglizzo \& Galetti 2003; Foglizzo et al. 2005; Ohnishi et al. 2005), or the stalled accretion shock instability (SASI) of Blondin et al. (2003) and Blondin \& Mezzacappa (2005), may be of significant importance for globally distorting the supernova shock during the shock revival phase and for accelerating the neutron star to velocities in excess of several hundred $\mathrm{km} \mathrm{s}^{-1}$ (Scheck et al. 2004, 2006). Here we have demonstrated that such an early non-radial, low-mode shock instability may also be of crucial importance for the observational appearance of a supernova, because it can provide the shock anisotropy which is responsible for the very strong Richtmyer-Meshkov instability that we observe at the $\mathrm{He} / \mathrm{H}$ interface. Judging from the results of our simulations, this latter instability also deserves much more appreciation by supernova modelers than it has received so far.

Although our simulations make use of a polar grid and the assumption of axisymmetry, a fact which we critically assess in Sect. 3.2, the development of the discussed low-mode instabilities and the corresponding explosion asymmetries is neither a numerical artifact, nor a consequence of nonconvergence of the simulations due to a lack of resolution, or even code deficiencies. The variability of the early explosion asymmetries found in the present study, and in the work of Scheck et al. (2006), is the result of an extremely nonlinear, chaotic growth of the initially imposed small random seed perturbations. No numerical convergence of simulations (in the mathematical sense) can be expected in such a situation, especially for morphological aspects like the shock deformation or the matter distribution in the unstable layer. Such aspects differ even between runs of exactly the same model, if these runs are carried out on different computers with slightly different 64 bit round-off behaviour. However, integral quantities like the explosion timescale and energy, or the neutron star mass, show little differences between simulations which make use of the same boundary conditions, in spite of large variations of the explosion geometry. Based on a huge set of models this is discussed in detail in Scheck et al. (2006). Therefore we do not expect that the findings presented here will be challenged by future simulations with higher grid resolution or different numerical schemes.

Furthermore, the occurrence and the final extent of the mixing due to the Richtmyer-Meshkov instability is fairly robust against the range of early explosion asymmetries that was explored in the present paper. All three low-mode models that we have computed for the Woosley et al. (1988) progenitor (i.e., models b18a, b18b, and b23a) show a comparable extent for the inward mixing of hydrogen and the outward mixing of metals in mass (for a comparison between models b18b and b23a, see Fig. 6). A larger sample of simulations is, however, required for a more reliable assessment of this issue.

The non-rotating models that we have presented here predict non-rotating neutron stars (unless an off-center kick imparts also a sizeable spin to the neutron star). This is in agreement with the fact that to date no pulsar has been found in SN 1987 A. However, this point could be satisfied equally well by a rotating neutron star with a weak surface magnetic field. Such an object would not produce significant pulsar activity, and its thermal cooling signature falls below the present detection limit. It remains to be seen in future simulations, though, whether rotating models can be constructed that are also able to reproduce the other observational items that we have summarized above.

A pulsar would, of course, also be lacking if a black hole had formed in SN 1987 A. This option, however, is quite unlikely for an $18-20 M_{\odot}$ progenitor and with the core structure of current progenitor models would require an unacceptably soft nuclear equation of state, which seems to be excluded by the recent detection of a neutron star with a well-measured mass of $2.1 \pm 0.2 M_{\odot}($ Nice et al. 2005).

While we are well aware of the fact that comparably well resolved three-dimensional models and detailed lightcurves, spectra, and polarization data still remain to be calculated, we think that already the present results provide support to the hypothesis that SN 1987 A was an explosion which fits into the framework of the neutrino-driven explosion paradigm, and that its understanding does not require the assumption of (more) controversial physics.

In the future we plan to run long-time simulations for type $\mathrm{Ib}, \mathrm{c}$ explosions, in which the $\mathrm{He} / \mathrm{H}$ interface is absent and the large anisotropy of the metal and/or helium core which we found in our models will be exposed to the observer from the outset. We believe that the global asymmetries imprinted on the ejecta by low-mode hydrodynamic instabilities in the neutrinoheated postshock layer may also offer an explanation of the large-scale anisotropy of the element distribution visible in the Cassiopeia A supernova remnant (Hwang et al. 2004). Our results in Figs. 8 and 9, and the results of Paper I, suggest that in this case the velocities of iron-group material can be expected to be even higher for a given energy of the explosion.

Acknowledgements. We are grateful to F.-K. Thielemann for supplying us with his rate library, and to F.-K. Thielemann and R. Hix for a copy of their nucleosynthesis code, which turned out to be very helpful for coding and testing our own routines. We would also like to thank S. Bruenn and S. Woosley for providing us with the initial data, S. Blinnikov and F. X. Timmes for discussions, and the anonymous referee and V. Utrobin for their constructive comments which have helped us to improve our paper. K.K., L.S. and H.-Th.J. were supported by the Sonderforschungsbereich 375 on "Astroparticle Physics" and the Sonderforschungsbereich-Transregio 7 "Gravitational Wave Astronomy" of the Deutsche Forschungsgemeinschaft. The work of T.P. was supported by the US Department of Energy under Grant No. B523820 to the Center of Astrophysical Thermonuclear Flashes at the University of Chicago. The simulations were performed on the IBM p655 of the Max-Planck-Institut für Astrophysik, on an SGI Altix 350 on loan to the ASC FLASH Center by SGI, on the NEC SX-5/3C of the Rechenzentrum Garching (RZG), and on the IBM p690 clusters of the RZG and the John von Neumann Institute for Computing, Jülich. We are especially grateful to the last two institutions for their support.

\section{References}

Bader, G., \& Deuflhard, P. 1983, Numer. Math., 41, 373

Blinnikov, S. I. 1999, Astron. Lett., 25, 359

Blondin, J. M., \& Mezzacappa, A. 2005, ApJ, submitted

[arXiv: astro-ph/0507181]

Blondin, J. M., Mezzacappa, A., \& DeMarino, C. 2003, ApJ, 584, 971

Bouchet, P., Phillips, M. M., Suntzeff, N. B., et al. 1991, A\&A, 245, 490

Bruenn, S. W. 1993, in Nuclear Physics in the Universe, ed. M. W. Guidry, \& M. R. Strayer (Bristol: IOP), 31

Bruenn, S. W., Raley, E. A., \& Mezzacappa, A. 2006, ApJ, submitted [arXiv: astro-ph/0404099]

Buras, R., Rampp, M., Janka, H.-T., \& Kifonidis, K. 2003, Phys. Rev. Lett., 90, 241101

Buras, R., Rampp, M., Janka, H.-T., \& Kifonidis, K. 2006a, A\&A, 447, 1049

Buras, R., Rampp, M., Janka, H.-T., \& Kifonidis, K. 2006b, A\&A, submitted [arXiv: astro-ph/0512189]

Burrows, A., Hayes, J., \& Fryxell, B. A. 1995, ApJ, 450, 830

Chandrasekhar, S. 1961, Hydrodynamic and hydromagnetic stability (Oxford: Clarendon)

Chevalier, R. A., \& Soker, N. 1989, ApJ, 341, 867

Colella, P., \& Woodward, P. R. 1984, J. Comput. Phys., 54, 174

Colgan, S. W. J., Haas, M. R., Erickson, E. F., Lord, S. D., \& Hollenbach, D. J. 1994, ApJ, 427, 874

Einfeldt, B. 1988, SIAM J. Num. Anal., 25, 294

Fassia, A., \& Meikle, W. P. S. 1999, MNRAS, 302, 314

Foglizzo, T. 2002, A\&A, 392, 353

Foglizzo, T., \& Tagger, M. 2000, A\&A, 363, 174 
Foglizzo, T., \& Galetti, P. 2003, in Proc. of the workshop, 3-D signatures in stellar explosions, June 10-13 2003, Austin, Texas, USA [arXiv: astro-ph/0308534]

Foglizzo, T., Scheck, L., \& Janka, H.-T. 2005, ApJ, submitted [arXiv: astro-ph/0507636]

Fryer, C. L. 1999, ApJ, 522, 413

Fryer, C. L., \& Warren, M. S. 2002, ApJ, 574, L65

Fryer, C. L., \& Warren, M. S. 2004, ApJ, 601, 391

Haas, M. R., Erickson, E. F., Lord, S. D., et al. 1990, ApJ, 360, 257

Hawley, J. F., \& Zabusky, N. J. 1989, Phys. Rev. Lett., 63, 1241

Herant, M. 1995, Phys. Rep., 256, 117

Herant, M., \& Benz, W. 1992, ApJ, 387, 294

Herant, M., Benz, W., Hix, W. R., Fryer, C. L., \& Colgate, S. A. 1994, ApJ, 435, 339

Hix, W. R., \& Thielemann, F.-K. 1996, ApJ, 460, 869

Hoffman, R. D., Woosley, S. E., Weaver, T. A., Rauscher, T., \& Thielemann, F.-K. 1999, ApJ, 521, 735

Hungerford, A. L., Fryer, C. L., \& Rockefeller, G. 2005, ApJ, 635, 487

Hungerford, A. L., Fryer, C. L., \& Warren, M. S. 2003, ApJ, 594, 390

Hwang, U., Laming, J. M., Badenes, C., et al. 2004, ApJ, 615, L117

Janka, H.-T., \& Müller, E. 1996, A\&A, 306, 167

Jennings, D. E., Boyle, R. J., Wiedemann, G. R., \& Moseley, S. H. 1993, ApJ, 408, 277

Kane, J., Arnett, W. D., Remington, B. A., et al. 2000, ApJ, 528, 989

Kifonidis, K., Plewa, T., Janka, H.-T., \& Müller, E. 2000, ApJ, 531, L123

Kifonidis, K., Plewa, T., Janka, H.-T., \& Müller, E. 2003, A\&A, 408, 621 (Paper I)

Kozma, C., \& Fransson, C. 1998, ApJ, 497, 431

Liou, M.-S. 1996, J. Comput. Phys., 129, 364

Liou, M.-S. 2000, J. Comput. Phys., 160, 623

Liou, M.-S., \& Steffen, C. J. 1993, J. Comput. Phys., 107, 23

McCray, R. 1993, ARA\&A, 31, 175

Meshkov, E. E. 1969, Izv. Akad. Nauk SSSR, Mekh. Zhidk. Gaza, 4, 151

Miles, A. R. 2004, Physics of Plasmas, 11, 5140

Mitchell, R. C., Baron, E., Branch, D., et al. 2001, ApJ, 556, 979
Müller, E. 1986, A\&A, 162, 103

Nagataki, S. 2000, ApJS, 127, 141

Nice, D., Splaver, E. M., Stairs, I. H., et al. 2005, ApJ, submitted [arXiv:astro-ph/0508050]

Ohnishi, N., Kotake, K., \& Yamada, S. 2005, ApJ, submitted [arXiv:astro-ph/0509765]

Phillips, M. M., Hamuy, M., Heathcote, S. R., Suntzeff, N. B., \& Kirhakos, S. 1990, AJ, 99, 1133

Press, W. H., Teukolsky, S. A., Vetterling, W. T., \& Flannery, B. P. 1992, Numerical Recipes in FORTRAN, The Art of Scientific Computing, Second Edition (Cambridge: Cambridge University Press)

Richtmyer, R. D. 1960, Comm. Pure Appl. Math., 13, 297

Ronchi, C., Iacono, R., \& Paolucci, P. S. 1996, J. Comput. Phys., 124, 93

Scheck, L., Kifonidis, K., Janka, H.-T., \& Müller, E. 2006, A\&A, submitted [arXiv:astro-ph/0601302]

Scheck, L., Plewa, T., Janka, H.-T., Kifonidis, K., \& Müller, E. 2004, Phys. Rev. Lett., 92, 011103

Shigeyama, T., \& Nomoto, K. I. 1990, ApJ, 360, 242

Socrates, A., Blaes, O., Hungerford, A., \& Fryer, C. 2005, ApJ, submitted [arXiv: astro-ph/0412144]

Spyromilio, J., Meikle, W. P. S., \& Allen, D. A. 1990, MNRAS, 242, 669

Suntzeff, N. B., \& Bouchet, P. 1990, AJ, 99, 650

Thielemann, F.-K., Nomoto, K. I., \& Hashimoto, M. 1996, ApJ, 460, 408

Thompson, T. A., Quataert, E., \& Burrows, A. 2005, ApJ, 620, 861

Timmes, F. X. 1999, ApJS, 124, 241

Timmes, F. X., \& Swesty, F. D. 2000, ApJS, 126, 501

Utrobin, V. P. 2004, Astron. Lett., 30, 293

Utrobin, V. P., \& Chugai, N. N. 2005, A\&A, 441, 271

Utrobin, V. P., Chugai, N. N., \& Andronova, A. A. 1995, A\&A, 295, 129

Wang, L., Wheeler, J. C., Höflich, P., et al. 2002, ApJ, 579, 671

Wilson, J. R., Mathews, G. J., \& Dalhed, H. E. 2005, ApJ, 628, 335

Woodward, P. R., \& Colella, P. 1984, J. Comput. Phys., 54, 115

Woosley, S. E. 1988, ApJ, 330, 218

Woosley, S. E., Pinto, P. A., \& Ensman, L. 1988, ApJ, 324, 466

Yamada, S., \& Sato, K. 1991, ApJ, 382, 594 
K. Kifonidis et al.: Non-spherical core collapse supernovae. II., Online Material p 1

\section{Online Material}




\section{Appendix A: The AUSM + flux}

We consider the construction of a numerical flux function for the system of Euler equations

$\frac{\partial \boldsymbol{U}}{\partial t}+\frac{\partial \boldsymbol{F}(\boldsymbol{U})}{\partial x}=0$

where

$\boldsymbol{U}=\left(\begin{array}{c}\rho \\ \rho u \\ \rho E\end{array}\right), \quad \boldsymbol{F}(\boldsymbol{U})=\left(\begin{array}{c}\rho u \\ \rho u^{2}+p \\ \rho u H\end{array}\right)$

$\rho, u$, and $p$ have their usual meanings,

$E=e+\frac{u^{2}}{2}=H-\frac{p}{\rho}$

is the (specific) total energy, $e$ the internal energy, and $H$ the total enthalpy. We shall only summarize the calculation of the first order accurate AUSM+ flux for 1D Cartesian geometry here. The extension of the method to higher orders of accuracy, curvilinear grids, and more than one spatial dimension is done using standard techniques, (see e.g. Colella \& Woodward 1984; and Liou 1996) and will not be explained in detail here.

The AUSM+ scheme of Liou (1996) is a sequel to the Advection Upstream Splitting Method (AUSM) that was introduced by Liou \& Steffen (1993). A central idea in these second generation flux vector split schemes is that the advective and acoustic waves of the system (A.1) describe two physically distinct processes and should be treated as such by the numerical scheme. To achieve this, the following ansatz is made in AUSM+ to calculate the numerical flux, $\boldsymbol{f}_{j+1 / 2}$, at the interface $j+1 / 2$ between zones $j$ and $j+1$

$\boldsymbol{f}_{j+1 / 2}=m_{j+1 / 2} a_{j+1 / 2} \boldsymbol{\Phi}_{j+1 / 2}+\left(0, p_{j+1 / 2}, 0\right)^{\mathrm{T}}$,

i.e. $\boldsymbol{f}_{j+1 / 2}$ is written as the sum of a convective and a pressure term. In Eq. (A.4) the quantities $m_{j+1 / 2}, a_{j+1 / 2}, p_{j+1 / 2}$ and $\boldsymbol{\Phi}_{j+1 / 2}$ are interface values of the Mach number, sound speed, pressure and the modified state vector $\boldsymbol{\Phi}=(\rho, \rho u, \rho H)^{\mathrm{T}}$, which contains the total enthalpy, $H$, instead of the total energy, $E$.

The scheme proceeds by accounting for the effects of rightgoing and leftgoing nonlinear waves (with eigenvalues $u \pm a$ of the flux Jacobian $\partial \boldsymbol{F}(\boldsymbol{U}) / \partial \boldsymbol{U})$ by a proper definition of the quantities $m_{j+1 / 2}$ and $p_{j+1 / 2}$. The effects of the linearly degenerate field with eigenvalue $u$ are finally taken care of by a simple upwind selection of $\boldsymbol{\Phi}_{j+1 / 2}$. The choice of $a_{j+1 / 2}$ mainly affects the resolution of shocks (and is discussed in more detail below). The formulae for $m_{j+1 / 2}, p_{j+1 / 2}, a_{j+1 / 2}$, and $\boldsymbol{\Phi}_{j+1 / 2}$ given by Liou (1996) guarantee upwinding, an exact resolution of stationary shocks and of both stationary and moving contact discontinuities. Furthermore they ensure the positivity of the scheme, and most important for the present application, the avoidance of the "odd-even-decoupling" and carbuncle phenomena in multidimensional applications (see Liou 2000 for details).

Liou's formula for the interface Mach number is

$m_{j+1 / 2}=\mathcal{M}^{+}\left(M_{j}\right)+\mathcal{M}^{-}\left(M_{j+1}\right)$,

where the contributions of the rightgoing and leftgoing waves have been denoted with superscripts "+" and "-", respectively. Here

$M_{j}=u_{j} / a_{j+1 / 2}, \quad M_{j+1}=u_{j+1} / a_{j+1 / 2}$ are left and right Mach numbers that are computed using the common interface sound speed $a_{j+1 / 2}$. The split Mach numbers $\mathcal{M}^{ \pm}$are given by the functions

$\mathcal{M}^{ \pm}(M)= \begin{cases}\frac{1}{2}(M \pm|M|), & \text { if }|M| \geq 1 \\ \mathcal{M}_{\beta}^{ \pm}(M), & \text { otherwise }\end{cases}$

where the $\mathcal{M}_{\beta}^{ \pm}$are defined as

$\mathcal{M}_{\beta}^{ \pm}(M)= \pm \frac{1}{4}(M \pm 1)^{2} \pm \beta\left(M^{2}-1\right)^{2}, \quad \beta=1 / 8$

(and we have corrected a misprint in the corresponding equation of Liou 1996). Similarly, the interface pressure is defined as

$p_{j+1 / 2}=\mathcal{P}^{+}\left(M_{j}\right) p_{j}+\mathcal{P}^{-}\left(M_{j+1}\right) p_{j+1}$,

where the split pressures are given by

$\mathcal{P}^{ \pm}(M)= \begin{cases}\frac{1}{2}(1 \pm \operatorname{sign}(M)), & \text { if }|M| \geq 1, \\ \mathcal{P}_{\alpha}^{ \pm}(M), & \text { otherwise, }\end{cases}$

with

$\mathcal{P}_{\alpha}^{ \pm}(M)=\frac{1}{4}(M \pm 1)^{2}(2 \mp M) \pm \alpha M\left(M^{2}-1\right)^{2}, \quad \alpha=3 / 16$.

The final upwind selection of $\boldsymbol{\Phi}_{j+1 / 2}$ can be written concisely in terms of the quantities

$m_{j+1 / 2}^{ \pm}=\frac{1}{2}\left(m_{j+1 / 2} \pm\left|m_{j+1 / 2}\right|\right)$.

With their help the numerical flux at interface $j+1 / 2$ can be expressed as

$\boldsymbol{f}_{j+1 / 2}=a_{j+1 / 2}\left\{m_{j+1 / 2}^{+}\left(\begin{array}{l}\rho \\ \rho u \\ \rho H\end{array}\right)_{j}+m_{j+1 / 2}^{-}\left(\begin{array}{l}\rho \\ \rho u \\ \rho H\end{array}\right)_{j+1}\right\}+\left(\begin{array}{l}0 \\ p_{j+1 / 2} \\ 0\end{array}\right)$.

What remains to be specified is the interface sound speed $a_{j+1 / 2}$. In principle the simple arithmetic average

$a_{j+1 / 2}=\frac{1}{2}\left(a_{j}+a_{j+1}\right)$

may be taken at the expense of slightly smearing shock waves. The formula of Liou (1996)

$a_{j+1 / 2}=\min \left(\tilde{a}_{j}, \tilde{a}_{j+1}\right)$

with

$\tilde{a}=a_{*}^{2} / \max \left(a_{*},|u|\right)$,

and the critical speed of sound, $a_{*}$, calculated via the isoenergetic condition for an ideal gas

$H=\frac{a^{2}}{\gamma-1}+\frac{1}{2} u^{2}=\frac{(\gamma+1) a_{*}^{2}}{2(\gamma-1)}$

gives sharper shocks. In particular it allows for exact resolution of stationary shocks (the generalization of (A.15) to more general equations of state, in which the ratio of specific heats, $\gamma$, is not a constant, is straightforward). Moving shocks are generally captured within one to two zones.

Numerical experiments confirm that the scheme is substantially less viscous than older flux vector splittings. In fact we have found the AUSM+ flux to be even less viscous than the Godunov flux (obtained from the exact solution of the Riemann 
K. Kifonidis et al.: Non-spherical core collapse supernovae. II., Online Material p 3

problem), which makes the former a somewhat less robust building block than the latter if used in higher order schemes to compute problems with complicated shock interactions (as e.g. the well-known blastwaves problem of Woodward \& Colella 1984). For the present application, however, in which we employed the AUSM+ flux only near a single strong shock (where furthermore the flattening algorithm of PPM reduced the spatial reconstruction to first order accuracy), the method worked well. 